\title{
THE EFFECTS OF INGROUP DEVELOPMENT IN A POST-MERGER JOINT-BRAND CONTEXT
}

Jay Newdick

\author{
A thesis submitted to Victoria University of Wellington \\ in fulfilment of the \\ requirements for the degree of \\ Master in Commerce and Administration \\ in Management
}

Victoria University of Wellington

2009 


\begin{abstract}
Mergers and acquisitions (M\&As) involve a complex relationship between two previously separate organisations. Social Identity Theory (SIT) has been applied to the study of M\&As as a way to better understand this relationship. To date, SIT literature has focused on developing the relationship between the merging organisations, in turn relinquishing the identity of the pre-merger organisations in favour of the new organisational identity. This research examines the constructs of the pre-merger groups, focusing on the pre-merger ingroups as a significant contributor to success in the post-merger environment.

In some M\&As, both pre-merger brands continue to operate simultaneously in the postmerger environment, and it is in this context that the ingroup plays an important role in post-merger integration. This research looks at ingroup development within a post-merger joint-brand context in order to assess the significance of ingroup identification within the post-merger environment. Ingroup development involves generating member identification with the pre-merger ingroup, rather than building identification with the post-merger organisation as a whole. Although there is literature to support the continuation of premerger ingroup identity in the post-merger environment, ingroup identification has generally been seen as a hindrance to the merger integration process.
\end{abstract}

A New Zealand based case study was examined to explore the significance of ingroup development in the post-merger context. The research looked into the effects of implementing an "ingroup development intervention" within the case study. The research design used forty semi-structured interviews to create a 'before' and 'after' assessment of the case study in order to gauge the effects of the ingroup development intervention. The findings of the research were analysed using thematic analysis, which was able to assess the change in participant perceptions over a period of time.

The findings showed that the ingroup development intervention resulted in a perceived reduction in status differences between the pre-merger groups, as well as a reported improvement in both ingroup and intergroup relations. The findings suggest that in a specified context, ingroup development can have a positive effect on the post-merger environment. The results of the research hold implications for both theorists and 
practitioners. The research provides intergroup theory with a greater understanding of ingroup identification and the extent to which it is effective within the post-merger environment. For practitioners, the research exhibits the value in post-merger organisations committing to long-term identity development for staff. 


\section{ACKNOWLEDGEMENTS}

I thank my parents, Graham and Debbie Newdick, who through their years of sacrifice have given me the opportunity to fulfil my potential. Your commitment to my success has been unwavering, and your example is a template for me to follow in the future. I also acknowledge my research supervisor Sally Riad, who throughout the research process has kept a high level of professionalism and commitment toward the research. I would like to recognise my fellow students, many of whom have been with me from the start of this process. I have enjoyed riding the highs and lows with you all, and may your success continue into the future. Finally, I would like to acknowledge my brothers and sisters at Davidic Centre. Your constant encouragement and support has been an aid throughout my journey; your fingerprint is on my process. 


\section{CONTENTS}

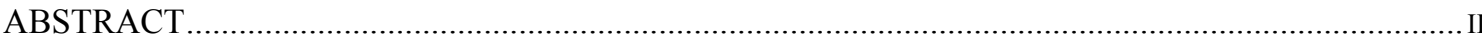

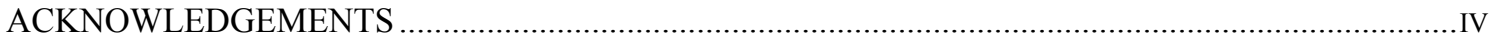

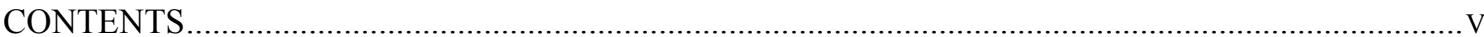

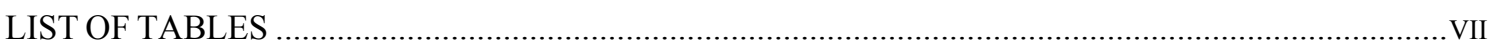

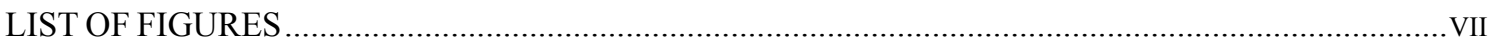

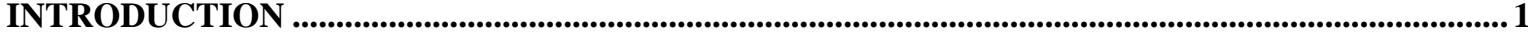

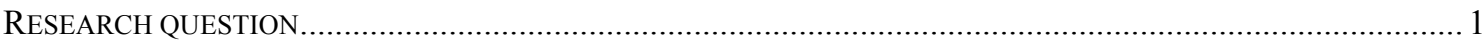

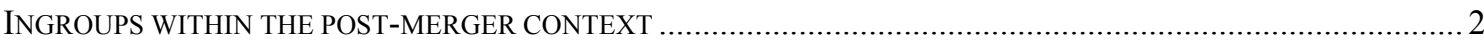

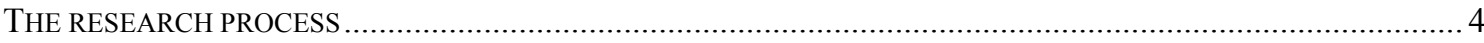

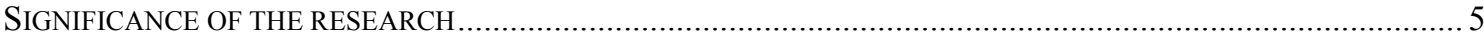

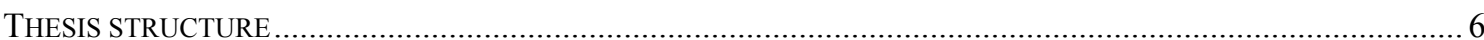

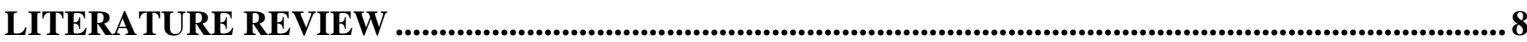

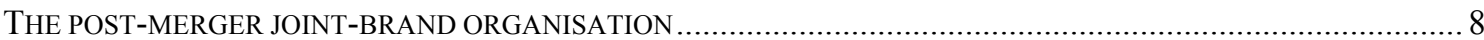

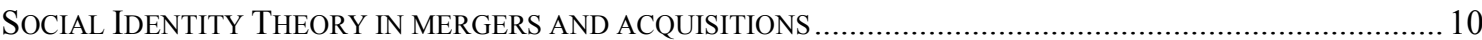

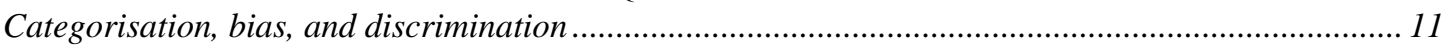

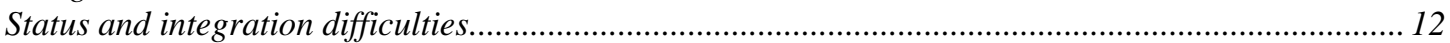

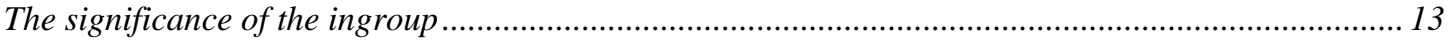

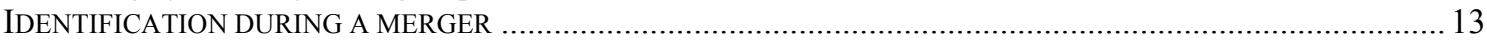

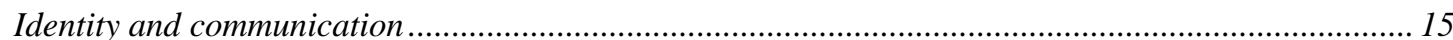

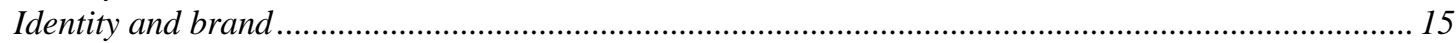

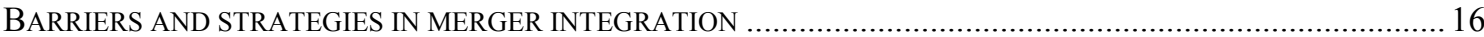

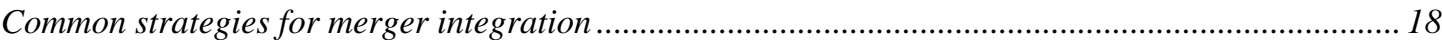

Revised merger approach: Considering the ingroup ..................................................................... 19

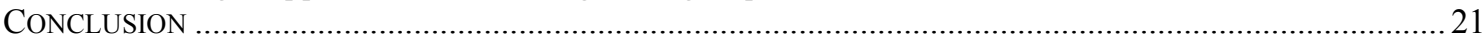

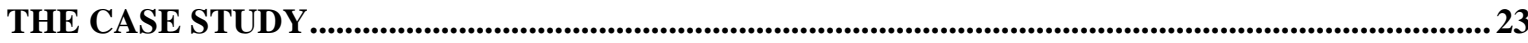

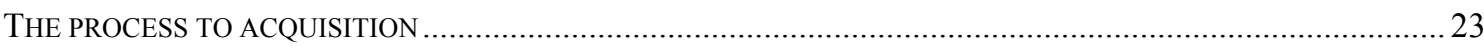

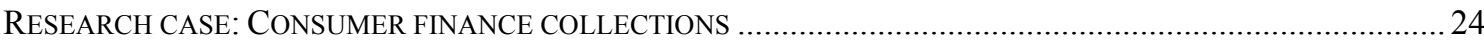

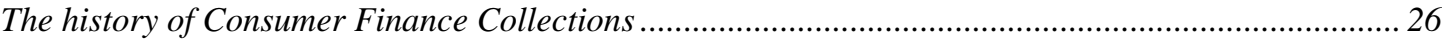

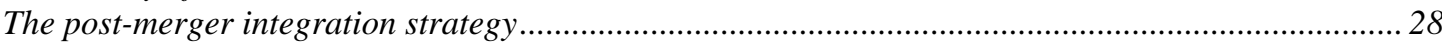

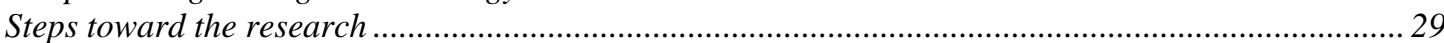

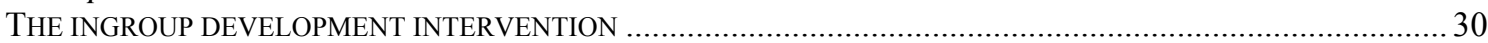

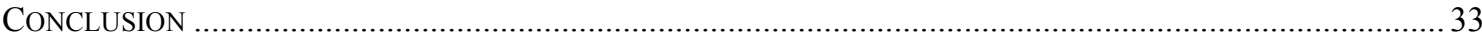

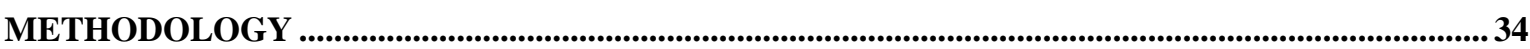

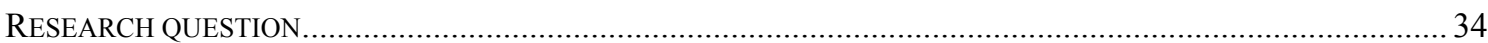

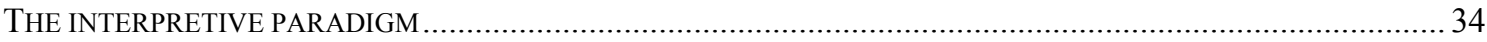

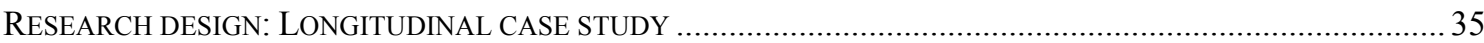

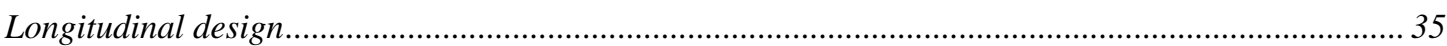

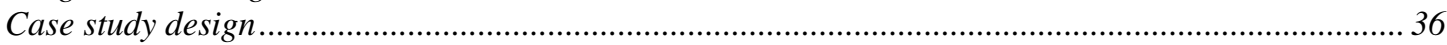

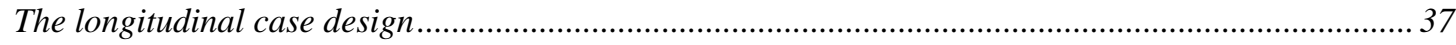

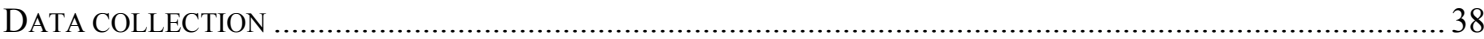

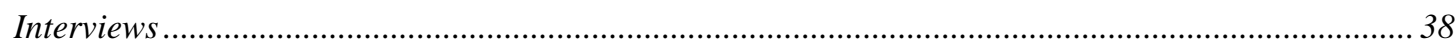

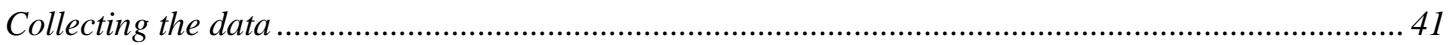

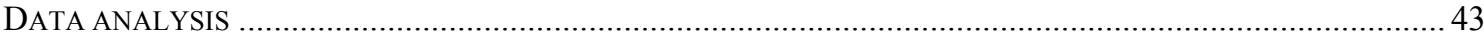

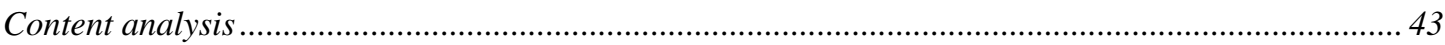

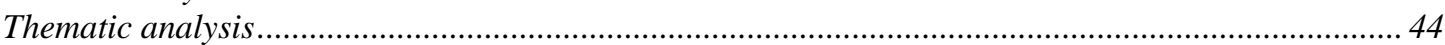

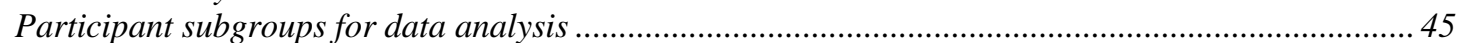

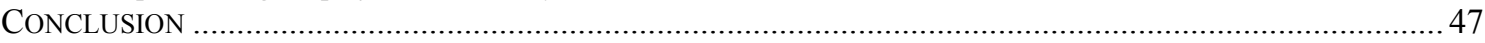

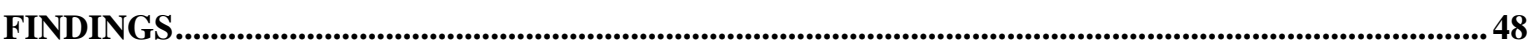

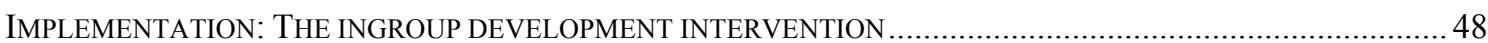

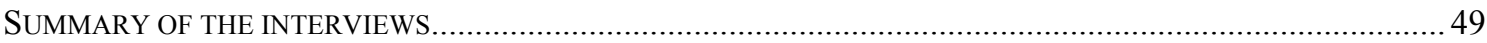

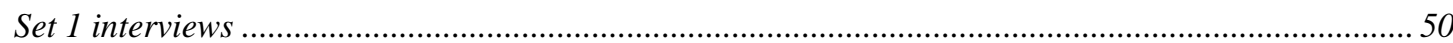

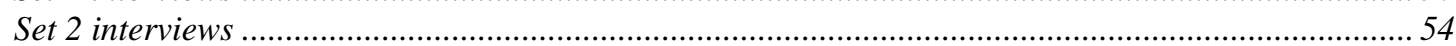

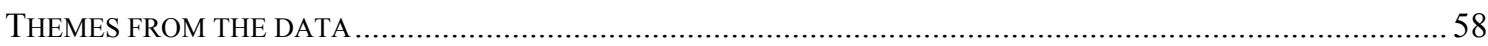




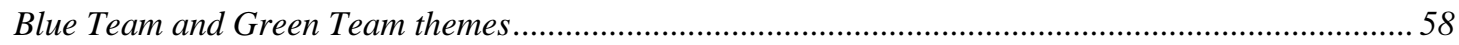

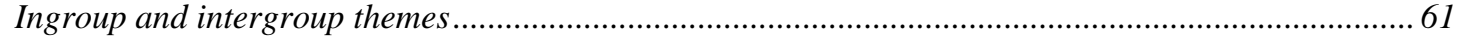

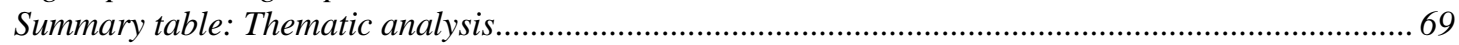

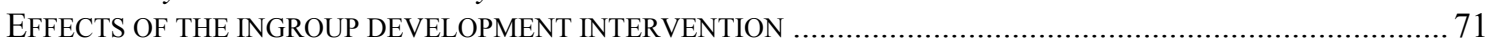

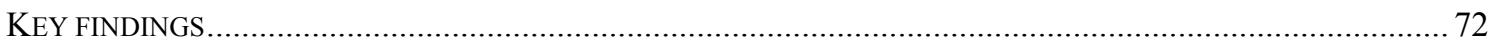

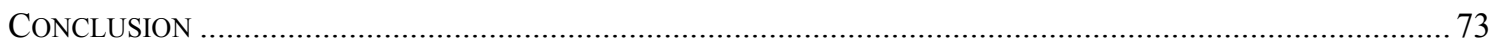

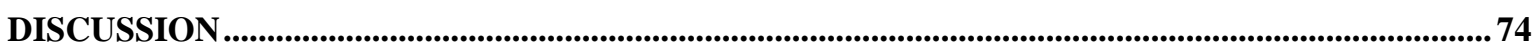

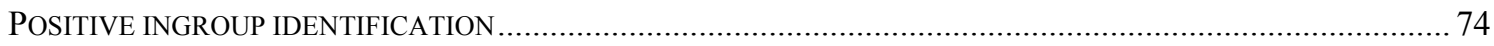

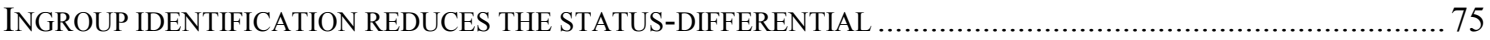

LOW STATUS-DIFFERENTIAL ALLOWS FOR INGROUP IDENTITY DEVELOPMENT ..........................................76

REDUCED PRE-MERGER IDENTITY RESULTS IN LOW ORGANISATIONAL IDENTIFICATION ............................ 77

REDUCED ORGANISATIONAL IDENTIFICATION ALLOWS FOR THE EFFECTIVE USE OF INGROUP INITIATIVES IN

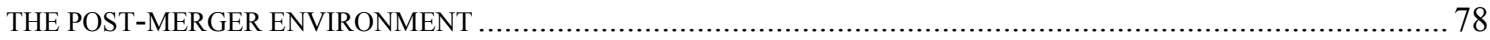

INTERGROUP DIFFERENTIATION IN THE POST-MERGER JOINT-BRAND ENVIRONMENT ................................ 79

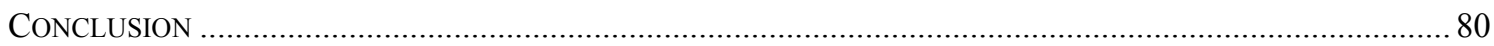

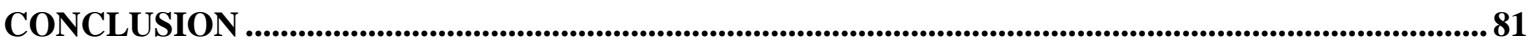

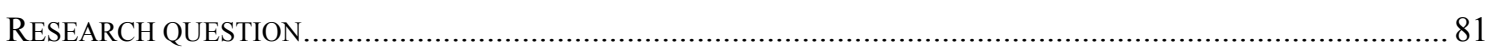

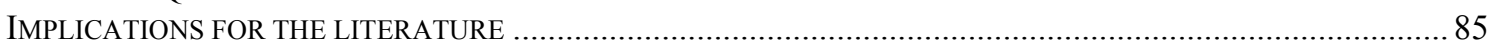

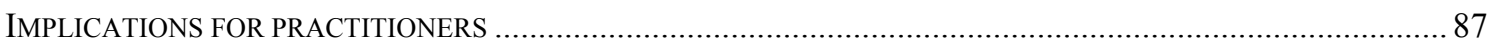

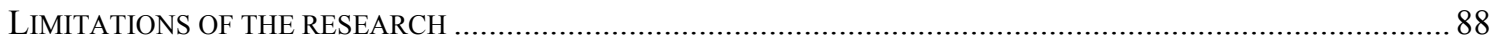

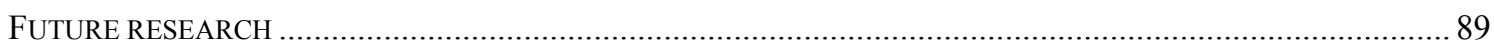

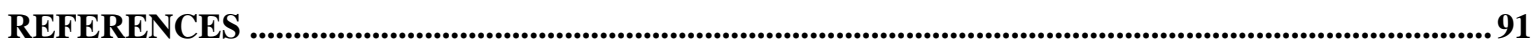

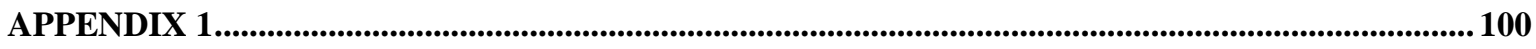

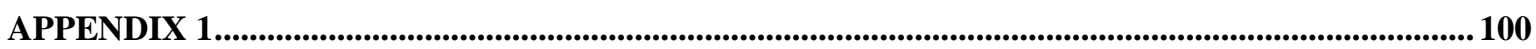

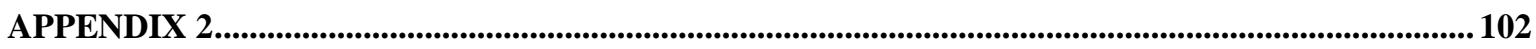

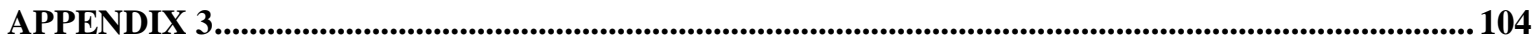




\section{LIST OF TABLES}

TABLE 1 NUMBER OF PARTICIPANTS AND ATTRITION RATE 41

TABLE 2 THEMATIC ANALYSIS SUMMARY TABLE 70

\section{LIST OF FIGURES}

FIGURE 1 ORGANISATIONAL STRUCTURE OF CONSUMER FINANCE COLLECTIONS 26

FIGURE 2 CASE STUDY LAYOUT PRE-INGROUP DEVELOPMENT INTERVENTION ........................... 31

FIGURE 3 CASE STUDY LAYOUT POST-INGROUP DEVELOPMENT INTERVENTION .......................... 32

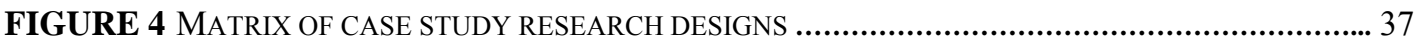

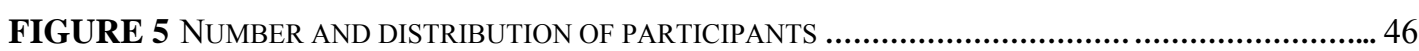

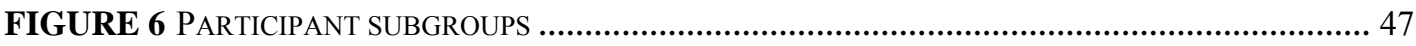

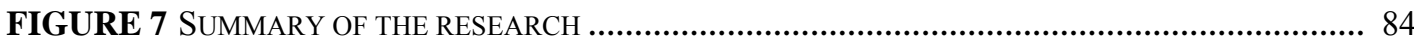




\section{Chapter 1}

\section{INTRODUCTION}

This research aims to develop understanding around ingroup identification in a postmerger context. The research adopts a Social Identity Theory (SIT) perspective, which states that individual identification is influenced by the groups of which an individual is a member. The study was conducted in a New Zealand based organisation which, for the purpose of this research, is named Blue Green Bank. Although Blue Bank purchased Green Bank in late 2003, both brands continued to operate simultaneously within the post-merger environment. This made for a unique business structure in that the branch network of Blue Bank and Green Bank remained separate, while the back office processing and call centre operations of Blue Bank and Green Bank were merged for efficiency purposes. The research involved implementing an "ingroup development intervention" within Consumer Finance Collections; one of the call centre operations in Blue Green Bank. Simultaneously, two sets of twenty interviews were undertaken to create 'before' and 'after' views of the case which, in turn, were used to assess the effects of the intervention. The findings report a perceived reduction in status differences between the pre-merger groups, as well as an improvement in the perception of ingroup and intergroup relations. These findings hold significance for SIT literature, as ingroup identification is often viewed as an inhibitor to post-merger integration. The findings also offer value for practitioners by underlining the effects of positive ingroup identification.

\section{Research question}

The research question for this study is: What are the effects of ingroup development in a post-merger joint-brand context? Here, the term "ingroup development" refers to building ingroup identification within an intergroup context. The research addresses a gap in SIT literature which stops short of examining the significance of ingroup identification during post-merger integration. 


\section{Ingroups within the post-merger context}

Mergers and acquisitions (M\&As) are popular activities in today's business environment, yet the majority of research indicates that most mergers are problematic (Fridolfsson \& Stennek, 2005). Financial or market factors are often cited as the cause of M\&A failure. However, results also show that a lack of M\&A success is commonly linked to human factors within the post-merger environment (Covin et al., 1996).

Mergers and acquisitions are two different business processes but, when studying the postintegration organisation, mergers and acquisitions are usually considered simultaneously. A merger is defined as the formal combination of two organisations (Prichett, 1987), whereas an acquisition is the transfer of the target company's resources to the acquirer (Peng, 2006). For the purposes of this research, both mergers and acquisitions are considered to be the combining of two previously separate organisations through the transfer of assets, operations, and management (Katsioloudes, 2006). This research focuses on what comes after the announcement of a merger, commonly known as post-merger environment.

In studying the post-merger environment Social Identity Theory (SIT) has been applied to better understand the merger process (Mottola et al., 1997). This research adopts an SIT perspective which is based on the premise that an individual's self-concept is determined within a social group (Hogg, 2006). There are at least two main social groups within a merger, each representing one of the pre-merger organisations. Traditional SIT research has focused on the relationship between these two groups which is known as the study of "intergroup relations". However, this research focuses on the pre-merger groups themselves, commonly known as the study of "ingroup dynamics". Ingroup dynamics is of increasing interest in SIT research (Tyler, 2000), and this research explores the significance of the ingroup in the post-merger organisation.

Post-merger integration literature has tended to favour intergroup relations over pre-merger ingroup development. This has been driven by the notion that merger integration is hindered because each organisation brings a different pre-merger identity to the newly formed organisation (Datta et al., 1990; Graves, 1981; Olie, 1994). In response to this, the literature suggests that pre-merger identities and culture should be relinquished in favour of 
a new organisational identity (Buono \& Bowditch, 1989; Cartwright \& Cooper, 1993; Gaertner et al., 1990; Giessner et al., 2006). This approach draws away from developing the pre-merger ingroups; instead it focuses on building organisational identification between the merging groups.

There has been hesitance to develop pre-merger ingroups in the post-merger environment because of the well-documented issues that arise from ingroup identification. The literature states that ingroup identification has a positive impact on ingroups, which includes generating positive relationships between ingroup members (Gaertner et al., 1996) that, in turn, better prepares a group for survival (Mullen et al., 1992). Although ingroup identification benefits the ingroup, at the same time it can trigger bias and discrimination towards outgroup members (Tajfel et al., 1971; Tajfel \& Turner, 1979). The negative outgroup orientations that are generated from ingroup identification can hinder the merger process and increase the cost of integration.

Due to the nature of ingroup identification, it is no surprise that ingroup development initiatives have had varying results in post-merger organisations. Previous studies have shown that maintaining a common ingroup identity strengthens post-merger identification with the superordinate group (Van Leeuwen et al., 2003). However, the same studies also showed that the continuation of pre-merger identity also strengthened intergroup bias between the merging groups. Despite the mixed results, there is literature which suggests that the continuation of pre-merger ingroup identification is a necessary part of the postmerger integration process (Van Knippenberg et al., 2002; Van Leeuwen \& Van Knippenberg, 2003; Van Leeuwen et al., 2003). These studies are built on the premise that the majority of perceived ingroup bias is not so much anti-outgroup behaviour as it is proingroup behaviour (Brewer, 1979). The literature also suggests that developing ingroup identity can have a positive effect on the intergroup environment, and that it is only circumstances of disadvantaged status that turn this into anti-outgroup orientations (Haunschild et al., 1994). Ingroup development has the potential to play a positive role in the post-merger organisation, but the depth of research in this area is limited. This study seeks to expand the understanding of ingroup dynamics within the post-merger context. 


\section{The research process}

This thesis sought to better understand ingroup identification through researching a New Zealand based organisation. For the purpose of the research, the case study organisation is given the name Blue Green Bank. Blue Green Bank had previously gone through a merger where the Blue Bank had acquired the Green Bank in 2003. What was unique about the merger was that both the Blue and Green brands were continued after the merger took place. I was originally employed at Blue Green Bank within the Consumer Finance Collections division, and the research began when I approached the management team about the possibility of Masters Research. At the time, management were wrestling with the issue of managing both the Blue and Green brands within the same organisational environment. From the perspective of Blue Green Bank, this research was an opportunity to better understand the dynamics of their operational context, while I viewed the case study as an opportunity to develop ingroup identification within a post-merger organisation. The first interviews for the research were conducted in July 2007, and these were followed by the implementation of the ingroup development intervention.

The research was based around the assessment of the effects of the ingroup development intervention. The ingroup development intervention sought to develop member identification within the separate Blue and Green teams. There were several initiatives that were used in the intervention, including increased brand activities, seating changes, team meetings, and performance tracking. The intervention increased the visual differentiation between each branded team and the level of communication within the teams, as well as educating each team about their respective brands. The initial phase of implementation took two months, and this was followed by ongoing brand and team meetings which continued for the entirety of the seven month research process. The goal of the research was to assess the effect that the ingroup intervention had had on the case study environment.

The methodology for the research was designed to capture the unique context of the case study, while at the same time assessing the effects of the ingroup development intervention. To do this, an in-depth analysis of the case study was performed, and the result of this shaped the reporting of the research findings. The research design itself was a longitudinal case study which involved conducting two sets of identical interviews, one 
before and one after the ingroup intervention. Each interview set involved twenty separate interviews, and the data from each set was compiled and compared to assess the change in perceptions across the period of the study. The research was analysed using thematic analysis.

The research revealed several key findings. To begin with, the findings revealed that the ingroup intervention developed a greater sense of member identification within the Blue and Green teams. This had several effects on the case study, the first being an improved perception of ingroup and intergroup relations. In addition to this, there was a perceived reduction in the status differences between the Blue and Green branded teams. The findings were important for both the theoretical basis of the research, and the case study organisation.

\section{Significance of the research}

The findings of the study contribute to the field of post-merger SIT research. The findings show that, in a specified context, ingroup identification can have a positive effect on both ingroup and intergroup relations. The research also shows that ingroup identification can help to reduce intergroup status differences. These results have implications for SIT research and intergroup theory, which predominantly portray ingroup identification as a hindrance to intergroup relations. This research is also important for post-merger studies, as the results showed that ingroup development can be a viable merger strategy in the postmerger organisation.

The findings of the research, and the research process itself, held significance for the case study organisation and practitioners in the field of post-merger integration. The interview process offered research participants the opportunity to express their views within a confidential environment, and for the organisation to receive valued feedback. The results provided greater understanding of the joint-brand environment, and tested tools that can be used to improve post-merger integration. 


\section{Thesis structure}

The thesis is organised into seven chapters including the introduction:

Chapter 2 - Literature Review:

This chapter reviews the relevant literature in the fields of M\&A and SIT research. The chapter begins by describing the post-merger joint-brand environment, and then explains the relevance of SIT to post-merger integration. The chapter proceeds to discuss the significance of identity in the post-merger organisation, and outlines how the ingroup can play a key role in the merger integration process. The chapter concludes by highlighting ingroup identification as the focal point for the remainder of the research.

\section{Chapter 3 - Case Study:}

The case study chapter describes Blue Green Bank, which is a New Zealand-based organisation formed when Blue Bank acquired Green Bank in 2003. The chapter begins by describing the acquisition process that took place, followed by an introduction of the business unit that was used as the case study for research. The chapter describes the history of the case leading up to the research, and then explains the ingroup development intervention that was used in the study.

Chapter 4 - Methodology:

The chapter describes the methodological approach that was used to assess the effects of the ingroup development intervention. The research design is a longitudinal case study, which focuses on a single case over an extended period of time. There were a total of forty interviews applied in two sets, one before and one after the ingroup development intervention was implemented. The chapter concludes by outlining the steps that were taken to analyse the data that was gathered from the interviews.

\section{Chapter 5 - Findings:}

The findings chapter assesses the effects of the ingroup development intervention on the case study. The chapter begins by describing the implementation process, followed by a thematic analysis of the first and second interview sets. The chapter concludes by summarising the main findings of the research, the most significant being a perceived 
reduction in status differences, and a reported improvement in both ingroup and intergroup relations.

Chapter 6 - Discussion:

The discussion chapter combines the results from the data analysis with the theoretical focus of the research. There are several areas covered in this chapter, which address the implications of the findings for areas of SIT and intergroup relations within the context of post-merger integration.

Chapter 7 - Conclusion:

The final chapter summarises the research and answers the research question. The chapter concludes by discussing the implications of the research for both the literature and practitioners. 


\section{Chapter 2}

\section{LITERATURE REVIEW}

This chapter begins by providing a definition of the post-merger joint-brand environment, followed by discussing the Social Identity Theory (SIT) perspective of merger integration. The chapter then reviews the role of identity within a merger, and discusses some of the challenges that are involved in M\&As. Next, it presents some of the common theoretical perspectives that surround merger integration, followed by recent literature that explores the significance of ingroup identification within the post-merger environment. The chapter concludes by highlighting the gap in SIT literature which tends to stop short of addressing the significance of pre-merger ingroup identification with the post-merger environment.

\section{The post-merger joint-brand organisation}

The forces of globalisation and the increased competition in the market place have meant that M\&As have now become central activities in the global economy (Bower, 2001; Fitzgibbon \& Seeger, 2002; Huang \& Kleiner, 2004; Trautwein, 1990). M\&As provide inherent growth potential for companies (Huang \& Kleiner, 2004; Katsioloudes, 2006; Pablo \& Javidan, 2002) through increased market power, revenue, and shareholder value (Gugler et al., 2003; Huang \& Kleiner, 2004; Langford \& Brown, 2004). Almost immediately, M\&A activity provides the opportunity to lower competition in a market (Perry \& Porter, 1985), and rapidly increase mass resource sharing and resource accumulation (Hespeslagh \& Jemison, 1991; Swaminathan et al., 2008). Potential value can also found within the integration processes itself (Hogan \& Overmeyer-Day, 1994), which can result in both cost reduction and revenue enhancement activities (Schweiger \& Lippert, 2005). Despite the lucrative opportunities that M\&A presents, there is no escaping the fact that M\&A success rates are deplorably low (Lynch \& Lind, 2002; Schweiger, 2002). It has been estimated that between fifty and seventy-five percent of M\&As do not meet their expected financial outcomes (Papadakis, 2007). Therefore, M\&A can either add or destroy value (Larsson et al., 2002), and the strategy itself can be considered as nothing less than a risky business process (Jarillo, 2003).

The post-merger phase is important to the overall success of a merger or acquisition. The significance of the post-merger environment is built on the premise that all value creation 
takes place after the acquisition (Haspeslagh \& Jemison, 1991), a process which research shows can last up to seven years (Stybel, 1986). The post-merger phase looks to generate efficiency gains around organisational processes (Hakkinen \& Hilmola, 2005) and often involves the combining of operations (Trautwein, 1990). The combining of operations includes procedural, physical, managerial, and socio-cultural integration (Shrivastava, 1986). Because of the significance of this phase of the merger, a body of literature has formed which is dedicated to the post-merger integration process (Birkinshaw et al., 2000). The literature in this field has changed and developed over time.

It was initially thought that pre-merger factors were the prime determinant for M\&A success. Early studies focused on the pre-merger structural and market factors which attributed to M\&A outcomes (Drucker, 1981; Lubatkin, 1983; Napier, 1989; Perry \& Porter, 1985; Salter \& Weinhold, 1978). This included theories which focused on the relatedness between the acquiring and the acquired firm (King et al., 2004). However, premerger factors themselves were unable to predict M\&A success (Shrivastava, 1986), and researchers began to understand that the integration process was the most important factor in realising M\&A benefits (Larsson \& Finkelstein, 1999; Hespeslagh \& Jemison, 1991). Attention then shifted to developing an understanding of the integration process itself.

Literature that focused on the integration process began to describe the complex difficulties that were associated with merging two different organisations. The integration process is defined as the combining of structural and cultural factors to form a functioning whole (Pablo, 1994). The combination process involves tension between radical change and the preservation of what is valuable in both the physical and cultural attributes of the premerger organisations (Meyer \& Lieb-Doczy, 2003). Researchers observed several common problems that were associated with the tensions in the merger process, which included anxiety and uncertainty, helplessness and rejection, withdrawal and avoidance, divided loyalties and conflicts over new values (Fulmer \& Gilkey, 1988). The complexities that were associated with the integration process steered the literature to look at the less tangible aspects associated with M\&As.

M\&A literature turned to explore the human factors associated with M\&As. These studies looked into the social, cultural, and psychological aspect of integration (Stahl \& Mendenhall, 2005). From this research there were two main fields of study that emerged: 
the organisational behaviour perspective, and the process perspective. The organisational behaviour perspective considers the cultural compatibility of merging organisations (Olie, 1994), and has a common concern for generating employee satisfaction and shared identity amongst the merging staff (Birkinshaw, et al., 2000). In contrast, the process perspective focuses on creating value through the ability to manage the post-merger process in an effective and profitable manner (Greenwood et al., 1994; Haspeslagh \& Jemison, 1991; Jemison \& Sitkin, 1986). Although different fields held different perspectives, it was commonly recognised that merger integration affects an organisation on both a structural (Villinger, 1996) and human level (Birkinshaw et al., 2000). Integration literature had therefore progressed to a place where it was able to engage with many facets of the postmerger integration process.

In classifying and describing types of mergers, the literature identified the joint-brand merger. A joint-brand merger is a form of collaborative merger (Napier, 1989), where merging organisations are comprehensively combined through the blending of operations. A collaborative merger can occur in what is called a "merger of equals" (Stahl, 2006), where two equal sized companies merge into one. This can result in what Basu (2006) describes as a "joint-brand" merger. A key characteristic of the joint-brand merger is that both pre-merger brands are kept in the post-merger organisation. Basu (2006) says that a joint-brand merger occurs because each pre-merger organisation has a strong presence amongst their target customers. The result is an organisation which operates two relatively autonomous brands under one corporate banner.

\section{Social Identity Theory in mergers and acquisitions}

SIT was birthed in the 1970s by Henri Tajfel through what was initially a European analysis of intergroup relations. The core basis of SIT is defined as “...a social psychological analysis of the role of self-conception in group membership, group processes, and intergroup relations" (Hogg, 2006, p. 111). SIT is based on the claim that people perceive the social world through social categories (Doise, 1978; Tajfel et al., 1971; Tajfel \& Turner, 1979), and that an individual's identity is derived from membership in a social group (Seo \& Hill, 2005). SIT states that the more a member relates to the group, the more the individual is governed by the group itself (Hogg \& Abrams, 1988; Tajfel \& Turner, 1986; Turner et al., 1987). This means that the group environment acts as a 
platform for the attitudes and behaviours of group members (Van Knippenberg et al., 2002).

SIT is one of Social Psychology's pre-eminent theoretical perspectives which have been rapidly absorbed into mainstream organisational literature (Brown, 2000). Within M\&A literature, SIT has been used to understand intergroup (Van Leeuwen \& Van Knippenberg, 2003) and ingroup dynamics (Tyler, 2000), the study of organisational processes (Hogg \& Terry, 2000), integration patterns and the impacts on employees (Mottola et al., 1997), the relationship between social identification and employee motivation (Haslam \& Ellemers, 2005), pre-merger status and post-merger identity (Giessner et al., 2006), and issues such as organisational citizenship (Dutton et al., 1994). The broad application of SIT to organisational research has created a unique perspective of the M\&A environment.

\section{Categorisation, bias, and discrimination}

Intergroup categorisation is a foundational theory of SIT. In SIT, a group is defined as three or more people who construe or evaluate themselves in terms of shared attributes that distinguish them from other people (Hogg, 2006). SIT states that individuals perceive people groups through social categories (Mullen et al., 1992), and they strive to maintain a positive social identity derived largely from the social groups they belong to (Tajfel $\&$ Turner, 1979). The categorisation of social groups and the desire of individuals to optimise their social identity is a primary source of bias and discrimination within an intergroup context.

Intergroup bias and discrimination emerges from the effects of social categorisation. SIT literature states that the mere perception of belonging to two distinct groups is enough to trigger intergroup discrimination (Tajfel et al., 1971; Tajfel \& Turner, 1979). Intergroup bias occurs when group members believe that their group is superior to other groups, and members of the ingroup are prepared to discriminate on this basis of this superiority, which is known as ingroup favouritism (Brown, 2000). Therefore, the process of social categorisation is the precursor to intergroup bias and discrimination. 


\section{Status and integration difficulties}

The process of socially categorising groups according to shared attributes leads to the emergence of status. Status is defined as the general prestige in which a group is held (Mullen et al., 1992). In any intergroup situation, the dominant group will usually enjoy a position of higher status. However, in many cases power and status can be mutually exclusive within a social structure (Lenski, 1984). A merger represents the joining of two different groups, so status differences often emerge through the integration process.

A merger between two previously independent organisations is likely to make the employees' pre-merger organisational membership salient. This means that a merger will accentuate intergroup status differences even if these differences are not reflected in the distribution of power (Giessner et al., 2006; Mullen et al., 1992). SIT states that people aspire to be in the high-status group (Tajfel, 1975), as membership in low-status groups fails to provide members with a positive social identity (Terry et al., 2001). Therefore, a significant portion of SIT literature has been devoted to explaining the diverse reactions of members of dominant and subordinate social groups (Brown, 2000).

SIT literature found that within a merger, members of the high-status group exhibit different behaviours from those of the low-status group (Terry et al., 2001). In the interests of preserving their positive social identity, the high-status group can discriminate or exclude those of the low-status group (Seo \& Hill, 2005). Tajfel and Turner (1979) suggest that to combat low social identification, members of the low-status group either disassociate from the low-status group, or take collective action to improve the perceived status of their group. Although social resistance can manifest itself in a number of different ways, the general reaction from the low-status group is resistance to change (Donnelly, 1992). The end result is an antagonistic environment, which involves power struggles and resource disputes between the two status groups (Olie, 1994). In summary, M\&As accentuate the status differences between merging groups which, in turn, can generate behaviours that hinder the integration process. 


\section{The significance of the ingroup}

The ingroup is an underpinning consideration within SIT and intergroup studies. Early studies of ingroup dynamics showed that ingroup members exhibited positive feelings towards each other, and negative attitudes toward other groups (Sumner, 1906). The desire to maximise social identification generates social categorisations (Tajfel \& Turner, 1979), the result being that ingroup members see each other as more unique and distinct than outgroup members; a process which generates positive social behaviour within the ingroup (Gaertner \& Dovidio, 2000). Research shows that the majority of perceived ingroup bias is not so much anti-outgroup behaviour as it is pro-ingroup behaviour (Brewer, 1979). However, circumstances of disadvantaged status can reverse this trend and result in antioutgroup orientations (Haunschild et al., 1994). The patterns observed through common ingroup behaviour impact the intergroup environment, and have specific implications for the merger integration process.

When considering the ingroup structure within M\&As, the major consideration is the continuation of pre-merger identity. Previous SIT research has found that changing premerger group identities is a cause of intergroup bias and resistance to change (Terry \& Callan, 1998). A merger does not always require group members to completely forfeit their old organisational identity (Van Leeuwen et al., 2003), and having a common ingroup identity has shown beneficial results, such as generating positive feelings towards common ingroup members (Gaertner et al., 1996). Previous studies have shown that the perceived continuation of pre-merger group identity strengthens the relationship between pre-merger identity and identification with the superordinate post-merger group (Van Leeuwen et al., 2003). However, the same studies also showed that the continuation of pre-merger identity also strengthened intergroup bias between the merging groups. Therefore, although it is clear that the continuation of ingroup identity affects the post-merger environment, the question of whether this effect facilitates or hinders the integration process is relatively unknown.

\section{Identification during a merger}

The topic of identity has always been of interest within M\&As. The merger process represents a blurring of identity boundaries (Empson, 2004), and M\&As effect all types of 
identification including organisational, professional, and work group identity (Seo \& Hill, 2005). A key strength of SIT is that it has restored coherence in the understanding of identification within the organisation (Ashforth \& Mael, 1989). The identification process plays a key role in the challenges which are faced in merger integration (Schweiger et al., 1987).

The degree to which one relates to the organisation is known as 'organisational identity'. Albert and Whetten (1985) argue that organisations have their own identity, which consists of the central, distinctive, and enduring characteristics of the organisation among its members. Bearing in mind that part of an individual's self-concept is made up of a social dimension, organisational identity refers to the extent to which the social dimension of an individual aligns with the distinctive attributes of the organisation (Hogg \& Terry, 2000). Organisational identity is therefore defined as:

...the perception of oneness with or belongingness to an organisation, where the individual defines him or herself in terms of the organisation(s) in which he or she is a member (Mael \& Ashforth, 1992, p.104).

Research has shown that positive organisational identification results in productive business outcomes. The benefits of achieving positive organisational identification are clear, as individual employees have shown increased motivation, especially if the company has a positive reputation (Melewar et al., 2006). Other positive effects include ingroup cohesion (Empson, 2004), positive organisational behaviour (Ashforth \& Mael, 1989; Dutton et al., 1994), and reduced staff turnover (Mael \& Ashforth, 1995). Positive organisational identification has also shown itself to be an informal means of managerial regulation (Alvesson \& Wilmott, 2002). Due to the benefits that are generated from positive identification, achieving this has become a priority for the post-merger organisation.

Generating positive organisational identification is a process commonly known as identity construction. Identity construction brings organisational members into a greater knowledge of the core values and central beliefs of an organisation (Scott \& Lane, 2000). Research shows that positive organisational identification is constructed through communicating the values and objectives of the organisation, as well as positively differentiating the 
organisation from other groups (Empson, 2004). Therefore, mediums which can communicate the identity of the organisation to its employees are valuable for constructing identity.

\section{Identity and communication}

Issues of identity and communication are closely linked. The literature states that corporate communications transmit the unique dimensions of an organisation, defining the distinctiveness of the group and promoting the corporate identity of the organisation as a whole (Balmer \& Dinnie, 1999). Within a merger environment, an increase in communication has been shown to foster integration (Shah, 2000) by significantly reducing employee uncertainty (Searle \& Ball, 2004). Alternatively, a lack of communication is linked to fears of loss of identity and culture (Schweiger et al., 1987). It is clear that communication is a necessity for building positive organisational identity, but the methods of communication can be diverse in nature.

The field of communication and identity building has been extended to include visual communication, which is associated with the transmission of images and symbols within an organisation. Image management communicates that which is central to the organisation (Dutton et al., 1994; Olins, 1995), and therefore visual techniques are seen as having an influence on identity and culture (Melewar \& Jenkins, 2002). In practical terms, visual communication involves manipulating the physical design of the office in order to communicate certain values (Melewar et al., 2006), as well as branding activities to connect the employees with the organisation (Basu, 2006). These are necessary tools in building positive organisational identification.

\section{Identity and brand}

Brand and marketing activity is usually linked to advertising (Mitchell, 2002). However, branding is now seen as having an intrinsic connection with employees (Basu, 2006; Ind, 2004; Mitchell, 2002). On a fundamental level, "brands are clusters of functional and emotional values resulting in promises about unique and welcomed experiences" (De Chernatony, 2006, p. xi). The brand is what creates differentiation in the heart and mind of the customer (Benson \& Foley, 2005), which results in customer loyalty and subsequent 
profits (Keller, 2000). Strengthening the relationship between the brand and the employee maximises the use of intellectual resources (Ind, 2004) and lowers the need for employee supervision (De Chernatony, 2006). Branding gives employees an understanding of the organisation, inspiration, and a description of the desired styles of behaviour (Gylling \& Lindberg-Repo, 2006). Branding structures are therefore said to have a clear impact on the identity of an organisation.

The brand plays a fundamental role in organisational identification. The intrinsic nature of the brand has a clear impact on the identity (Balmer, 1998; Nandan, 2005; Pruzan, 2001) and culture (Dinnie, 2005) of an organisation. Branding proliferates organisational identity through the communication of the organisation's core values, which in turn gives employees the opportunity to understand and relate to the organisation (Gylling \& Lindberg-Repo, 2006). The opportunity to relate to the organisation will result in social identification within members, "As staff learn to identify closely with the values and objectives of the organisation, their organisation's identity will come to form an integral part of their self-concept” (Empson, 2004, p. 7). Therefore, an organisation's branding has an influence on the social identification of it members.

\section{Barriers and strategies in merger integration}

The literature has pinpointed pre-merger identity and culture as drivers of the peoplerelated problems that are experienced during integration (Graves, 1981; Hogg \& Terry, 2000; Marks \& Mirvis, 2001; Zaheer et al., 2003). The literature has developed several strategies which are used to address pre-merger identity and culture in the post-merger environment. The following section explains this view, and then presents an alternative perspective which suggests that pre-merger identity and culture can play a positive role in the post-merger environment (Van Knippenberg et al., 2002; Van Leeuwen \& Van Knippenberg, 2003; Van Leeuwen et al., 2003). The alternative perspective suggests that the ingroup can be used as a means to positively harness pre-merger identity and culture in the post-merger organisation. 


\section{Pre-merger identity and culture}

The literature states that the merger process is potentially problematic when each organisation brings a different identity and culture to the merger (Datta et al., 1990; Graves, 1981; Olie, 1994). As previously stated, an individual's identity is their selfconcept, which is constructed from both individual and social characteristics (Hogg, 2006). Within an organisational environment, SIT states that the more salient, stable, and internally consistent the character of the organisation is, the greater this character will be internalised within the individual's personal identity (Ashforth, 1985; Ashforth \& Mael, 1989). The internal consistency of the character of the organisation is often called the organisational culture, which relates to the common ideas, beliefs, and meanings which are taken for granted, but are necessary for continuing organisational activity (Alvesson, 2002). In a merger, each merging group brings a different identity and culture to the merger, commonly known as pre-merger identity and culture (Giessner et al., 2006). It is pre-merger identity and culture which the literature says shapes the people-related issues that are experienced during integration.

The literature states that the existence of pre-merger identity and culture potentially constrains the merging of two organisations into one new entity (Graves, 1981; Hogg \& Terry, 2000; Marks \& Mirvis, 2001; Zaheer et al., 2003). The accentuation of pre-merger identity and culture elevates the status differences which exist between merging groups (Giessner et al., 2006; Mullen et al., 1992), which in turn creates an "us versus them" scenario (Zaheer et al., 2003). An "us versus them" scenario can generate several adverse effects between groups that hold different degrees of status for example the low-status group can feel threatened (Terry \& O'Brien, 2001), and the high-status group can feel superior (Marks \& Mirvis, 2001). This results in reduced levels of post-merger identification from the low-status group (Van Knippenberg et al., 2002), and discriminative behaviour from the high-status group (Brown, 2000; Tajfel \& Turner, 1979). Pre-merger identity and culture are therefore considered a barrier to merger integration, and the literature recommends that both elements are reduced in the post-merger environment (Buono \& Bowditch, 1989; Cartwright \& Cooper, 1993; Gaertner et al., 1990; Giessner et al., 2006). 
Part of reducing pre-merger identity and culture is the elimination of intergroup distinctiveness. On a foundational level, distinctiveness between groups potentially contributes to bias and discrimination (Tajfel et al., 1971; Tajfel \& Turner, 1979). SIT states that merger activity accentuates pre-merger organisational membership, which then increases intergroup differences and consequently affects intergroup relations (Mullen et al., 1992). A merger is seen as the re-categorisation of two different social groups into one new group (Van Knippenberg et al., 2002). Therefore, the literature suggests strategies which eliminate intergroup distinctiveness in order to develop a unified approach.

\section{Common strategies for merger integration}

Based on the assumption that pre-merger identity, culture, and intergroup distinctiveness are potentially constraining to merger integration, the literature has put forth several merger strategies which aim to reduce pre-merger identity and culture within the postmerger environment. The following are three examples of such strategies:

A merger of equals: A "merger of equals" is a merger process which elevates the principle of equality for merger integration (Stahl, 2006). This is designed so that “...each merger partner would have the same status within the new company and would bring an equal degree of their pre-merger identity into the merger" (Giessner et al., 2006, p. 341). Equalising status and pre-merger identity in the post-merger environment would reduce the possibility of intergroup bias and discrimination (Tajfel et al., 1971; Tajfel \& Turner, 1979).

The third culture approach: M\&A literature state that creating a new culture in a merger can assist the integration process. Research done by Cartwright and Cooper (1993) on a wide range of industries suggests that within a merger of equals, a key determinant of successful integration depends on the ability to create a unique third culture. The third culture approach involves a process of relinquishing pre-merger cultures in favour of the culture of the new organisation (Buono \& Bowditch, 1989). This results in the reduction of pre-merger identification (Zaheer et al., 2003), which reduces intergroup bias through recategorising the two previously separate organisations into one single entity (Gaertner et al., 1990). 
Contact hypothesis: SIT states that intergroup differences are the source of intergroup bias and discrimination (Tajfel et al., 1971; Tajfel \& Turner, 1979). Early SIT research states that intergroup differences can be reduced through using "contact hypothesis". Initially developed by Allport (1954), "contact hypothesis" suggests that increasing intergroup contact eliminates intergroup misperceptions and allows group members to identify intergroup commonalities. This, in turn, improves intergroup attitudes (Brown, 2000) and reduces intergroup prejudice (Green, 2008).

These are three examples of strategies which the literature suggests can be used to eliminate pre-merger identity and culture within the post-merger environment. The literature supports the premise that pre-merger identity and culture can hinder merger integration. However, evidence suggests that both factors play a significant and often positive role in the post-merger organisation.

\section{Revised merger approach: Considering the ingroup}

\section{Pre-merger identity and culture}

Research shows that pre-merger identity and culture "lingers" in the post-merger environment (Buono \& Bowditch, 1989; Vaara, 2003). This can occur even within a merger of equals, as pre-merger identity is enhanced through merger integration (Zaheer et al., 2003). The prevalence of pre-merger identity meant that integration strategies began to allow for levels of pre-merger identification within the post-merger organisation (Giessner et al, 2006). However, research found that the existence of pre-merger identity could facilitate a sense of continuity of identity, leading to positive identification with the postmerger organisation (Van Leeuwen et al., 2003). Findings such as this began to facilitate questions around the common assumption that pre-merger identification and culture hinder the merger integration process.

Another area of interest is around the theory that intergroup distinctiveness leads to bias and discrimination, and whether this assumption is applicable to all merger contexts. Many mergers are collaborative mergers which involve a high level of combination and integration (Napier, 1989). A collaborative merger can also be a merger of equals (Stahl, 2006), where each company brings an equal degree of pre-merger identity to the newly 
formed organisation. This structure can be a joint-brand environment where each of the pre-merger brands continue to exist in the post-merger organisation (Basu, 2006). The literature shows that branding acts as a source of organisational identification, communicating the distinctiveness of the organisation to both customers and employees (Dutton et al., 1994; Gylling \& Lindberg-Repo, 2006; Olins, 1995). The literature also states that if members of each brand are to positively identify with the organisation, the distinctiveness of their brands must be evident (Hogg \& Terry, 2000) and frequently communicated (Empson, 2004). These actions would generate distinctiveness between merging groups, which suggests that distinctiveness may be a necessity within the postmerger joint-brand organisation.

\section{Considering the ingroup}

A common trend in post-merger strategies is to equalise the merger and to diminish premerger identification. This has proven challenging, as research states that creating a merger of equals is difficult due primarily to the fact that most mergers are not truly equal (Cartwright \& Cooper, 1996; Van Oudenhoven \& De Boer, 1995). Achieving equalisation is also difficult because mergers are shown to increase the salience of pre-merger organisational membership (Terry \& O'Brien, 2001). Even in an organisation that seeks to establish positive social identities, differences based on skill are enough to generate status differences between merging groups (Mullen et al., 1992). Strategies such as the "third culture approach" and "contact hypothesis" seek to develop a new organisational culture and identity. However, research shows that a significant source of resistance in a merger is generated through disrupting the pre-merger identities (Terry \& Callan, 1998). There are several challenges that arise when attempting to reduce pre-merger identification and culture, and the literature has begun to look for alternative integration strategies.

Mainstream literature has not promoted pre-merger ingroup identification due to the belief that this will generate intergroup bias within the post-merger environment. Foundational intergroup theory states that within an intergroup environment, distinctiveness between ingroups will trigger intergroup discrimination (Tajfel et al., 1971; Tajfel \& Turner, 1979). However, research shows that the majority of perceived ingroup bias is not so much antioutgroup behaviour as it is pro-ingroup behaviour (Brewer, 1979). The earliest recordings of ingroup bias are by Sumner (1906), who coined the term ethnocentrism to refer to the 
positive evaluation of the ingroup in relation to the outgroup. Although ingroup bias may primarily result in positive ingroup behaviour, the literature shows that circumstances of disadvantaged status can reverse this trend and result in anti-outgroup orientations (Haunschild et al., 1994). This suggests that in the appropriate context, pre-merger ingroup identification can have a positive effect on the intergroup environment.

There is a growing body of literature which states that maintaining pre-merger ingroup identity is a valid approach to merger integration (Van Knippenberg et al., 2002; Van Leeuwen \& Van Knippenberg, 2003; Van Leeuwen et al., 2003). On a foundational level, having a common ingroup identity generates positive ingroup relationships (Gaertner et al., 1996). In addition to this, the ingroup bias which is generated from the continuation of premerger identification is seen to improve the cohesiveness of the ingroup to better equip it for survival (Mullen et al., 1992). In summary, the literature shows that maintaining a common ingroup identity is a viable post-merger integration strategy.

\section{Conclusion}

The post-merger organisation is a complex environment which is centred on issues of identification. The literature showed that post-merger integration is commonly seen as the dissolution of pre-merger identities in favour of the new organisational identity (Haunschild et al., 1994). However, recent literature has re-evaluated pre-merger identification, and alternative literature has emerged which states that pre-merger ingroup identification can play a positive role in the post-merger organisation (Van Knippenberg et al., 2002; Van Leeuwen \& Van Knippenberg, 2003; Van Leeuwen et al., 2003). Although the literature has identified the potential benefits of pre-merger ingroup identification, the topic itself remains relatively unexplored.

This research will focus on the potential for ingroup development within the post-merger organisation. The literature showed that ingroup identification plays an important role in ingroup development (Mullen et al., 1992). However, this is rarely explored in the postmerger environment, as ingroup identification can cause intergroup bias and discrimination (Tajfel et al., 1971; Tajfel \& Turner, 1979). Previous research has focused on the continuation of pre-merger ingroup identification within a controlled environment (Van 
Leeuwen et al., 2003), but there are few studies within post-merger organisations, especially those which operate in a joint-brand context. 


\section{Chapter 3}

\section{THE CASE STUDY}

In 2003, Blue Bank purchased Green Bank to form the New Zealand-based organisation Blue Green Bank Limited. Blue Green Bank Ltd is a wholly-owned subsidiary of the Melbourne-based organisation Blue Banking Group. In 2003, Blue Bank and Green Bank were two of the five major banks competing in the New Zealand market, and the acquisition heralded the formation of New Zealand's biggest bank. Although analysts remained positive about the prospects of the acquisition, the task of integrating two previously separate banks lay ahead.

\section{The process to acquisition}

Prior to the acquisition, Blue Bank and Green Bank each held iconic positions in the New Zealand market. Commonly known by its light blue logo, Blue Bank was the first bank to be established in New Zealand when a branch was opened in Wellington in 1840. The Australian owned multinational expanded within the New Zealand market by continual growth in institutional and commercial banking. Blue Bank was in competition with Green Bank, who opened its first branch in New Zealand in 1873. The Green Bank expanded mainly within rural, personal, and small banking within New Zealand. The rural positioning of the organisation in conjunction with several key sponsorship activities made the Green Bank logo an icon within the New Zealand market.

Before the acquisition in 2003 both organisations had distinct business models and held different positions in the New Zealand market. The structure of Blue Bank was considered to be product focused, while Green Bank was described as having a customer centric approach. Green Bank's strategy led it to hold the highest customer service rating of all the banks in New Zealand, while Blue Bank had one of the lowest. These differences had an impact on the performance of each organisation. The Green Bank had been the bestperforming bank in New Zealand for the last ten years, while results showed that the Blue Bank had been losing market share. In the New Zealand market, Blue Bank had around fifteen percent market share, whereas Green Bank held around twenty-five percent. When 
the banks merged in 2003, the Blue Bank decided to maintain the Green Bank brand, thus establishing a joint-brand organisation.

Blue Bank's decision to keep Green Bank presented a unique business model, as both banks were owned by the same organisation, yet they continued to operate autonomously in the New Zealand market. Within the newly merged Blue Green Bank, the branches and other customer-facing functions would be kept separate and operate autonomously from one another. In contrast, the back-office functions would involve the blending of the operations of both organisations to achieve efficiency gains. This meant that although the customer-facing staff would not be directly affected by the merger, the back-office staff faced the prospect of a significant integration process.

\section{Research case: Consumer finance collections}

The case study is a back-office department of Blue Green Bank named Consumer Finance Collections (CFC). The details of the case study are listed below:

Operations:

CFC is a call centre environment where staff members (known as 'collectors') contact Blue and Green Bank customers to collect monies owing on credit card and personal loan products. The business unit is open from $8 \mathrm{am}$ to $8 \mathrm{pm}$, and is only closed on Sundays and some public holidays.

Teams and brand structure:

Although the acquisition occurred in 2003, CFC was not established until 2005 when the Blue Credit Card Collections Team (Blue Team) was created to join the Green Credit Card Collections Team (Green Team) in Wellington, New Zealand. Two further teams were added to CFC, as the Blue and Green Personal Loan Collection Teams (Blue Personal Loans and Green Personal Loans) were moved to CFC in 2007.

Although CFC is an unbranded business unit, teams within CFC are grouped by brand. For example the credit card side of CFC has both a Blue Team and a Green Team; each operate autonomously and are managed by separate Team Leaders. The personal loan business also 
has a Blue team and a Green team, but because of the smaller size of the personal loans operation there is only one Team Leader for both groups.

Size:

At the beginning of the study in July 2007 there were around thirty-five full time employees working in CFC. After the seven month duration of the research there were approximately fifty full time employees in CFC.

Management structure:

There is one senior manager who overlooks eight business units within Blue Green Bank, and one of these business units is CFC. Within CFC itself, there is one operational manager and four team leaders.

Team structure for research:

There are four core teams in CFC. The longest serving team is the Green Team, followed by the Blue Team who joined the Green Team in 2005. These teams represent the credit card side of the business, and the majority of the research participants come from these two teams. There are also two teams that represent the personal loan side of the business; these are the Blue Personal Loans Team and the Green Personal Loans Team. These teams are smaller than the credit card teams, and for the purposes of the research they will be referred to as the joint Personal Loans Team. The organisational structure of CFC is shown in Figure 1: 


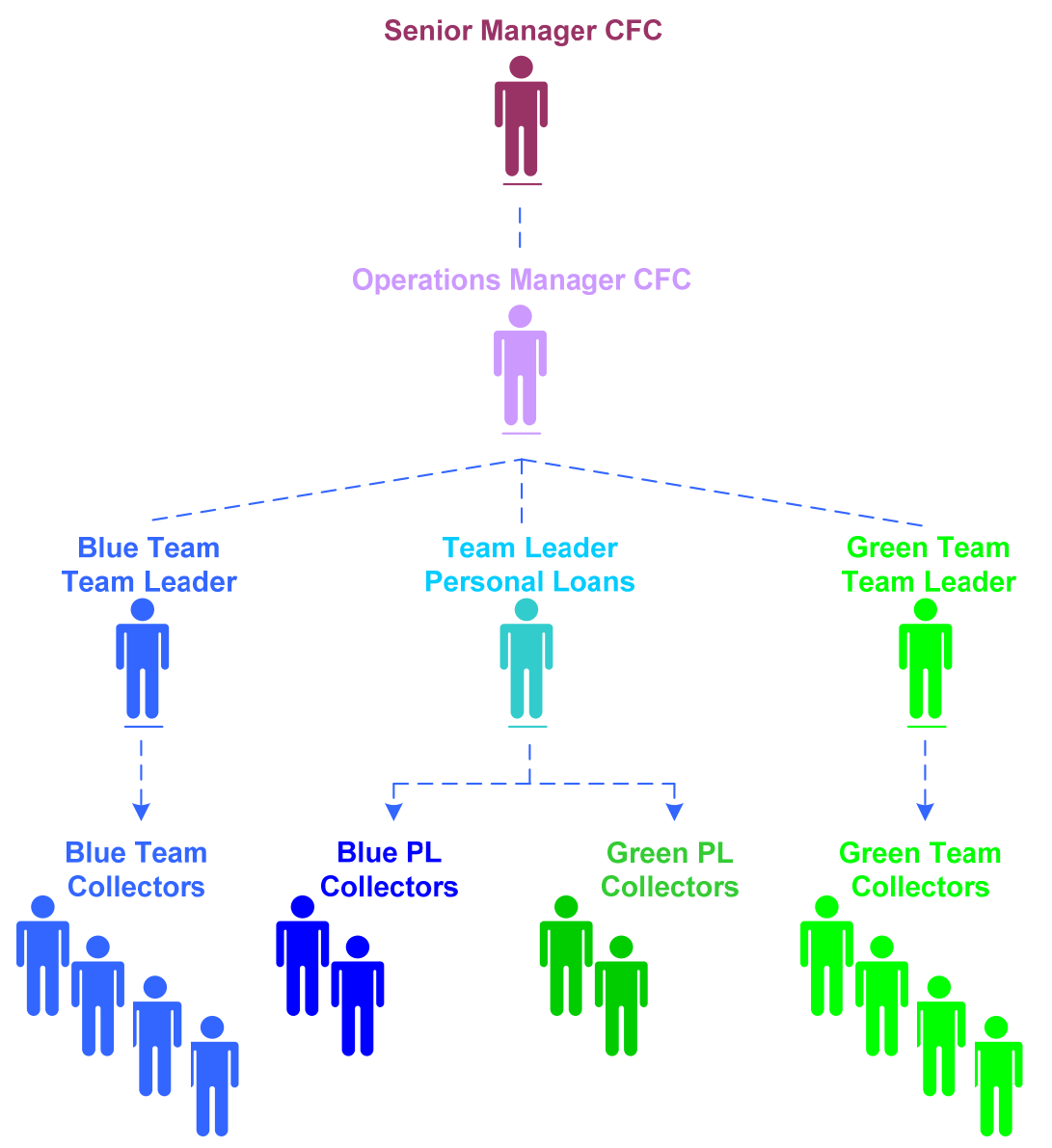

Figure 1: Organisational structure of Consumer Finance Collections

\section{The history of Consumer Finance Collections}

CFC was opened in 2005 when the Blue Team was moved from Melbourne (Australia) to join the Green Team who was based in Wellington (New Zealand). There were three management positions available within the newly established business unit. The operational manager position was filled by the previous Team Leader of the Melbourne based Blue Team. The Blue Team Leader position was filled by a previous team member of the Melbourne based Blue Team, while the previous Green Team Leader retained their position in the Wellington based operation. CFC continued for around a year and a half before two further collection teams were added to the business in early 2007. The additional teams were the Blue Personal Loans Team and the Green Personal Loans Team, which were moved from other areas of the bank in order to consolidate collection 
operations. The addition of the two personal loan teams increased the number of staff in CFC from twenty-five to thirty-five full time employees (approximate).

Each team within CFC had its own unique history. The Green Team was the only team that existed within New Zealand before the acquisition in 2003. Many of the staff who still worked in the team had been in collections prior to the acquisition. This team had the most experience out of all CFC teams. In contrast to the Green Team, the Blue Team had no premerger experience due to its formation in 2005. Besides one employee who switched from the Green Team to join the new Blue Team, all staff in the Blue Team were new to CFC. Over time, it became evident that the Blue Team was the most effective collection team in CFC, due primarily to system advantages that the Blue Bank had over the Green Bank. The two personal loan teams also held a unique history. The teams were transferred from another business unit, which involved a volatile transition process. The teams initially had around five staff members each, and they were assigned one overarching Team Leader. Due to system advantages, the Blue Personal Loans Team was established quickly in comparison to the Green Personal Loans Team.

The structure of the office in CFC was segmented according to the branded teams. Each team performed the same operational function, but there were differences in terms of systems, strategies, and customers. What this meant was that branded team members needed to be seated together. Although teams were seated together, the seating was not clearly differentiated, which made it difficult to tell where one team started and where another ended. There were also inconsistencies with the seating, as some staff members were seated among other branded teams.

In the months leading up to the research, there were several sizable human resource changes that impacted CFC. The first of these changes was the announcement of a new collections team that was to be established in the department. This team was called the Recoveries Team, and the roles in the team were on a higher pay band than that of a normal collector. A lot of the work that was to be performed by the Recoveries Team was already being done by the Green Team. Consequently, when the Recoveries Team was announced, the Green Team was faced with the prospect of losing part of their job role to higher paid staff. This was a strong source of contention for the Green Team leading up to the start of the research. 
In addition to the announcement of the Recoveries Team, there were several other changes that effected CFC leading up to the research. The first was the appointment of two new Team Leaders. The first Team Leader was to head the newly established personal loan teams, and the second was to take over the role for the Green Team. The other major change in the area was a seating change that was done in order to make room for the growing personal loan teams. There were several errors and subsequent corrections that had to be made with this change, and again this was a point of contention for staff in the lead up to the start of the research.

\section{The post-merger integration strategy}

Blue Green Bank exhibited two different merger strategies for managing the operations of the organisation. The branch network underwent an "extension merger" (Napier, 1989), where the operations remained relatively untouched. This was evident in the fact that both the Blue and Green branch networks continued to operate autonomously in the post-merger environment. In contrast, the merger strategy that was used for the back-office operations is known as a "collaborative merger" (Napier, 1989), which involves the comprehensive combining of operations to achieve operational efficiencies. In Blue Green Bank the collaborative merger was seen through combining Blue and Green back-office operations into single business units. Although the operations were combined, the Blue and Green Teams remained distinct from one another, as each brand held different operating systems. In summary, the overall merger strategy of the organisation was described as the "two dining rooms, one kitchen approach', whereby the 'two dining rooms' represented the separate Blue and Green Branch networks, and the 'one kitchen' represented the jointbrand back office operations such as CFC.

Within CFC, there were several merger integration strategies that were used. The merger was essentially a "merger of equals" (Stahl, 2006), which meant that both the Blue and Green brands had an equal level of power within the organisation. In addition to equalising power, CFC sought to establish a new third culture within the post-merger environment. This process involves relinquishing pre-merger identity and culture in favour of the new identity and culture of the post-merger organisation (Buono \& Bowditch, 1989). Within CFC, a "one bank" approach was adopted. However, there was a lack of visual material to support the new approach. This meant that the office environment was relatively bland, as 
neither the Blue/Green brands, nor the "one bank" approach was visually represented. To facilitate the "one bank" approach, senior management initiated Consumer Finance performance updates on a monthly basis. These meetings involved teams from both brands and they gave staff a formal update of the position of the organisation, while awarding top performers within the department. From an SIT perspective, these meetings eliminate intergroup misperceptions, and establish intergroup commonalities between the merging parties (Brown, 2000).

\section{Steps toward the research}

I was originally employed in CFC as a part-time Collections Officer. The research was initiated in early 2007 when I approached the senior manager of CFC about the possibility of conducting research. We discussed the status of $\mathrm{CFC}$, and the senior manager highlighted the difficulty that occurred in trying to manage two different brands operating in the same organisational environment. It was decided that a new business strategy would be implemented within CFC, and that I would conduct 'before' and 'after' interviews to assess the effects of the strategy. Within the case study, the new business strategy was called the "Brand Enhancement Initiative", and it was portrayed as a strategy that would restore the Blue and Green brands. For the purposes of the research, the business strategy represents the "ingroup development intervention". The implementation would involve several key actions that would be carried out by the operational manager and Team Leaders within CFC.

For the purpose of conducting the research, the senior manager gave full access to CFC. Full access included support from the operational manager and Team Leaders within the business, as well as a presentation (conducted by the senior manager and myself) to all the staff within CFC. The presentation explained the research and my role as a researcher. Once this material was presented to the staff, I was shifted into a new position within the bank which was outside of $\mathrm{CFC}$, at which time the ingroup development intervention was implemented and the research began. 


\section{The ingroup development intervention}

The purpose of the ingroup development intervention is to facilitate ingroup identification within the case study. Building ingroup identification means facilitating member alignment with the distinctive attributes of their branded team, rather than with the distinctive attributes of the organisation as a whole. To build ingroup identification, the intervention engages in identity construction, which brings ingroup members into a greater knowledge of the core values and central beliefs of their branded team (Scott \& Lane, 2000). The literature states that identification can be built by using several mediums which include: communication (Balmer \& Dinnie, 1999), visual tools (Olins, 1995), and branding (Balmer, 1998; Nandan, 2005; Pruzan, 2001).

To facilitate ingroup identification, the ingroup development intervention seeks to develop two key areas within the case study. The first area is the level of intergroup differentiation. Intergroup differentiation is the degree of distinctiveness between the Blue and Green teams within the case study. Due to the fact that organisational identification represents the alignment of members to the distinctive characteristics of the group, both the Blue and Green teams must express greater distinctiveness in order to facilitate increased member identification. The second area of development is the level of ingroup communication, as greater ingroup communication seeks to foster greater ingroup alignment, therefore facilitating ingroup identification. Both intergroup distinctiveness and ingroup communication are therefore important areas of focus for the ingroup development intervention.

The ingroup development intervention began implementation in July 2007 and remained in place until March 2008. The key steps that were involved in the intervention are listed below:

Action 1 - Seating and branding:

Before the research began, some staff members were not seated with their assigned team. Also, there was no branding displayed in the office, which meant that there was no visual differentiation between the branded teams. The seating layout before the research began is shown in Figure 2: 

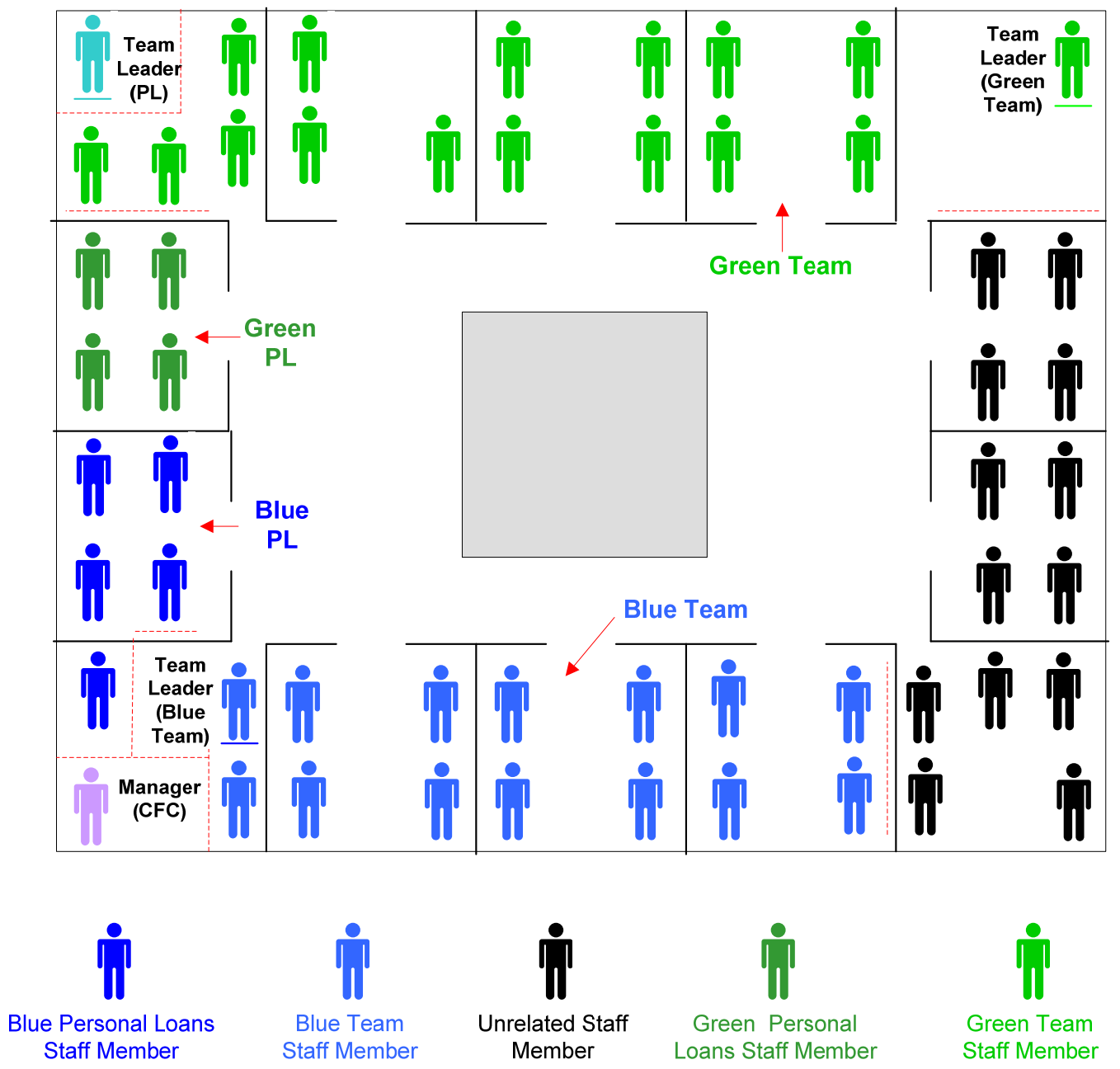

Figure 2: Case study layout pre-ingroup development intervention

The ingroup development intervention shifted the office environment to a symmetrical layout. The Manger of CFC and the Team leader of Personal Loans were placed at the centre of the office, and the branded Team Leaders were placed among their respective teams. All seats on the right hand side of the Manager were given to the Blue teams, and all seats to the left were given to the Green teams. The office was also branded, which meant that Blue posters occupied the Blue side of the office and vice versa. The layout of the office after the ingroup development intervention is shown in Figure 3: 


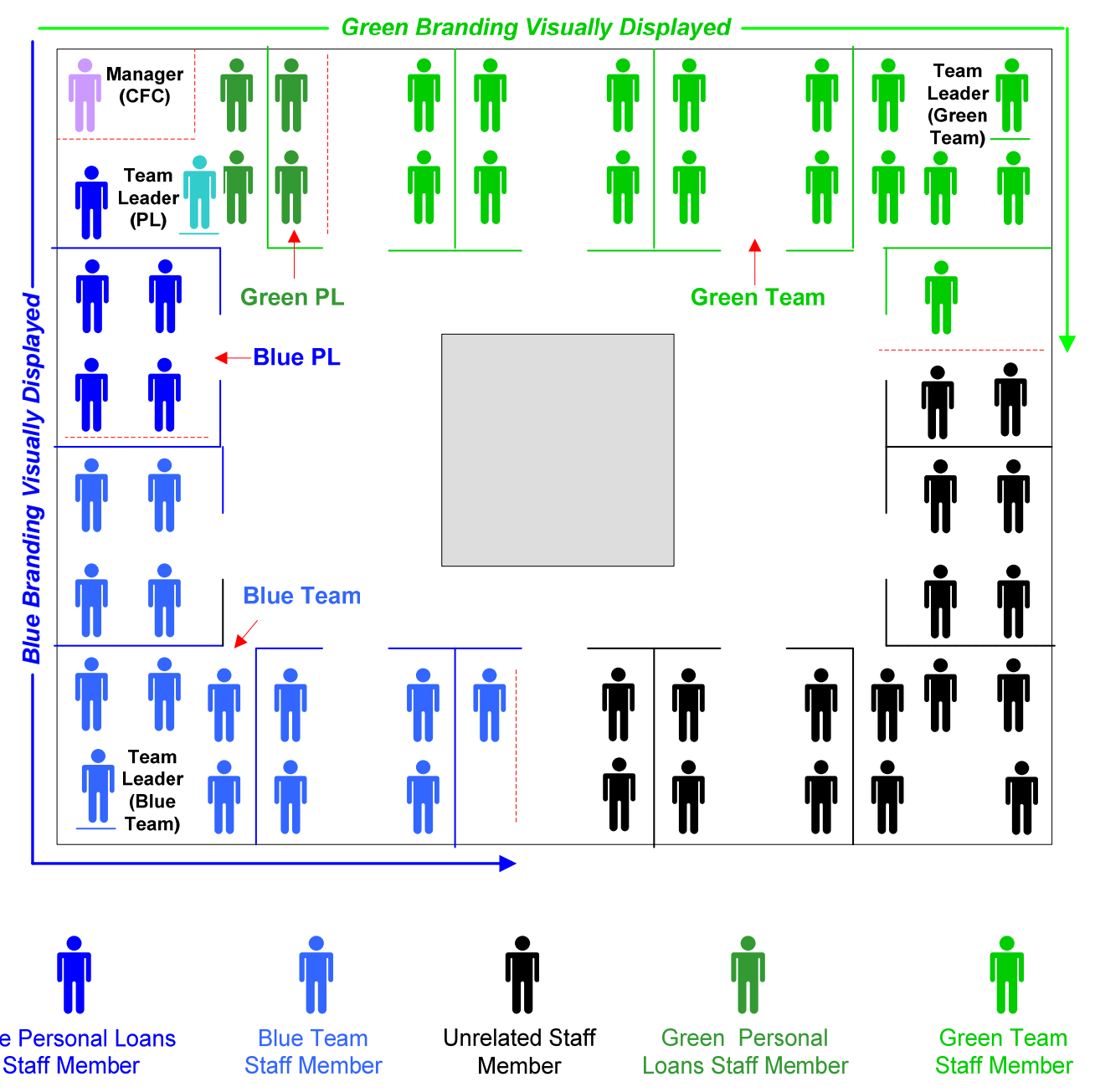

Figure 3: Case study layout post-ingroup development intervention

Action 2 - Restoring fortnightly team meetings:

The four teams within CFC previously had regular fortnightly team meetings, but in the six to eight months prior to the research these meetings had been forfeited due to the increased workload within CFC. A key part of the intervention was restoring these meetings, which would involve the Team Leader sharing and discussing relevant information.

Action 3 - Monthly brand meetings:

This was a new initiative to CFC and it involved, for example, combining the Green teams from $\mathrm{CFC}$ to discuss recent brand updates. There were separate meetings for both the Blue and Green teams, which would run for around twenty to thirty minutes. The meetings were conducted by the operational manager of CFC. 
Action 4 - Distributing brand material

Once the intervention was announced, brand material was distributed to all staff members. The Green staff received Green money boxes, pens, and drink bottles. The Blue staff received the same Blue branded products, with some staff also receiving branded note pads.

Action 5 - Team-based performance charts

The Team Leaders established a system for visually tracking the progress of their teams. A whiteboard was assigned to each team, and the team leader selected several key performance measures which were displayed on the whiteboards.

\section{Conclusion}

CFC had a history that was shaped by the Blue Bank's acquisition of Green Bank in 2003. Knowledge of this history shaped the methodological strategy that was used for the research, and this provided contextual value for the data that was gathered and analysed. For the research, the ingroup development intervention was the initiative that provided the basis for inquiry. The effects that were observed from implementing the intervention provided valuable data that was used to inform the SIT and intergroup theory that surrounded the study. 


\section{Chapter 4}

\section{METHODOLOGY}

The research involves qualitative inquiry based on a longitudinal case design. The research question is presented, followed by a description of the interpretive platform that guided the study. The chapter will then explain the major parts of the methodology including the longitudinal case design, the data collection method, and the data analysis process.

\section{Research question}

The research involved implementing the ingroup development intervention within the case study. The purpose of the research design is to gauge the effects that the intervention had on the case study. To support the question that guided the overall research, the following sub-question was used to guide the analysis of the data:

What are the effects of this ingroup development intervention in the post-merger jointbrand context?

The research question has two important dimensions. The first is the "ingroup development intervention", and the second is gauging the effects within the case study environment. The intervention itself has been described in the Case Study Chapter, which, from a theoretical perspective, is designed to develop ingroup identification within the branded teams in CFC. This will be done by implementing several changes within the organisation, each aimed to develop greater ingroup differentiation and communication in order to facilitate ingroup identification. In terms of assessing the effects of the intervention, the research will focus on changes that occur on the level of ingroup and intergroup dynamics.

\section{The interpretive paradigm}

"Be careful how you think; your life is shaped by your thoughts." (Proverbs 4:23)

Each piece of academic research is usually guided by a foundational paradigm of thinking. A paradigm is defined as "...a set of basic beliefs that deals with ultimates or first 
principles" (Guba \& Lincoln, 1994, p. 107). These beliefs and principles ultimately define the direction of the research as a whole. For this research, the interpretivist paradigm most closely describes the principles that guided the study.

The interpretive paradigm regards reality as being individually constructed, and claims that human affairs are inextricably involved with the intensions, goals, and purposes of the individual (Gage, 1989). In the field of academic research, the interpretive paradigm views research as being guided by the set of beliefs and feelings which are held by the researcher (Denzin \& Lincoln, 1994). In addition to this, the interpretive paradigm states that the researcher and the research subject cannot be separated because the research subject is essentially subjective, and it is the only source of knowledge and reality for the researcher (Guba, 1990). Interpretive research does not value broad generalisations, but seeks to produce highly descriptive and contextual results (Johnson \& Onwuegbuzie, 2004). The principles of the interpretivist paradigm have shaped the current study.

\section{Research design: Longitudinal case study}

A longitudinal case study is a research design which combines the longitudinal and case study approaches to research. This section will describe both the longitudinal and case study strategies, concluding with a description of the design that is used for this research.

\section{Longitudinal design}

A longitudinal study is research which is conducted over an extended period of time. Theodorson and Theodorson (1969) define longitudinal research as the "study of individuals over a period of time" (p. 236). Longitudinal research also includes at least two stages of data collection (Schulsinger et al., 1981). Having two stages of data collection is

a dimension that is unique to the longitudinal design, giving the researcher an ability to generate a 'before' and 'after' observation of the research subject (Buhler et al., 1973).

Although the longitudinal design was founded in quantitative research, it has been adapted for use in qualitative studies. In place of its empirical application, qualitative longitudinal methods have been developed to capture the benefits of the longitudinal design, while allowing for descriptive and contextual qualitative content (Ruspini, 2002). Holland et al (2006) describes the advantages of qualitative longitudinal research as: 
- The ability to study transition which privileges the subjective, context, and complexity of the case;

- Understanding the impact of changing circumstances;

- Understanding how changes result from particular interventions;

- The evaluation of specific policies or programmes aimed at achieving specific outcomes;

- Developing insight into developmental processes such as the construction of identities;

- Providing a realistic understanding of causality in social and cultural dimensions.

The development of the qualitative longitudinal method has given social researches the ability to intervene in social environments, and to assess the effects of the intervention through rich qualitative descriptions.

When collecting longitudinal data the most common research design is known as a prospective longitudinal design. The prospective design is often called a 'panel study', as the same panel of participants are studied in-depth over an extended period of time. The prospective design gives the researcher an ability to highlight differences and changes between one time period and another, to identify "sleeper effects" (hidden influencing forces) within the study, to explain changes in terms of other characteristics, and to give contextual definitions of the data (Ruspini, 2002). The prospective design is therefore a robust qualitative method which gives unique insight into the process, change, and continuity of phenomena over a period of time (Elliott et al., 2008).

\section{Case study design}

Case study research is commonly used in qualitative inquiry. The case study approach has several defining factors, the first of which is the unit of measure. Case study research is the study of a single or small number of units also known as "cases" (Hartley, 1994). Case study research is unique in that it examines contemporary phenomenon in its real-life context, and it is often renamed as "field work" because the case study involves inquiry in the natural environment (Stake, 2005). By definition, the case study is a naturalistic form of inquiry, in which the research subject and the context of the research cannot be separated (Yin, 2003). A case study design can include the following characteristics: a qualitative method, a holistic and thick (comprehensive) examination of a phenomenon, 
the gathering of evidence which is naturalistic, and data which cannot be separated from its context (Gerring, 2007).

The case study represents a strategy which is commonly used to assess complex social issues which other research approaches cannot address. In conjunction with qualitative longitudinal research, the case design has been used to contribute to the fields of psychology, sociology, political science, and business, through developing greater understanding of the individual, group, or community. Within each of the listed fields, the demand for case study research has been generated from the desire to understand complex social phenomena, rather than the need to make population generalisations (Yin, 2003). Case study research is effective in these areas because it allows for detailed contextual analysis of the cases, which can be used to generate knowledge around a new topic or to extend the previous understanding of a real-life situation (Spring, 1997). The ability to study complex social phenomena has meant that the design has been used to assess societal or community issues, to evaluate the effectiveness of programs, or to understand the implications of policies (Mabry, 2008).

\section{The longitudinal case design}

The longitudinal design and case study design are compatible methods within qualitative inquiry. In a similar fashion to longitudinal research, case study research holds a distinctive place in evaluative studies, which adopts an exploratory approach towards assessing the effects of interventions on an environment (Yin, 2003). Gerring (2007) depicts the longitudinal design within case study research:

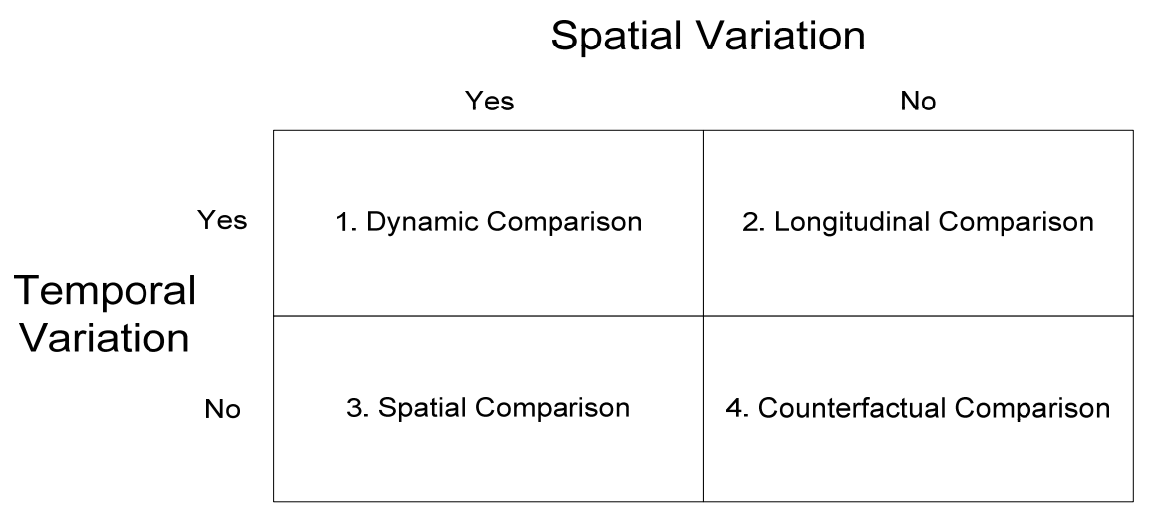

Figure 4: Matrix of case study research designs 
As shown above, the longitudinal design holds a place within case study research based on the prerequisite that there is no variation in the case or the boundaries of the study (spatial variation), while there is variation in terms of time period (temporal variation). This research will assess the same case study, with the only variation being the time period at which the different stages of data collection are taken. The research will also be a single case study; a dimension that both the longitudinal and case study designs allow for. Although multiple cases are often used to increase the ability to generalise results, it is commonly accepted within both longitudinal and case study research that a single case study is more detailed than a multiple case approach (Schulsinger et al., 1981). This research will analyse a single case using the longitudinal method.

\section{Data collection}

The following section outlines the data collection process. This begins by describing the interviews that were used for data collection. After this is a brief description of how interviews add to the research, followed by a detailed explanation of how the interview questions were generated from the relevant literature. The section concludes by describing the process in which the data was collected, which details the specific challenges that were encountered within the case study.

\section{Interviews}

As described by Stake (2005), a case study design is not necessarily a methodological choice as much as it is a choice to study a certain subject. This statement is true for the current study, as the case itself determined the research design and data collection process. From the outset, the research was a single case study, and the decision to implement the ingroup development intervention was the point of value for both the researcher and the organisation. The best way to assess the effects of the intervention was to have the ability to view both 'before' and 'after' snapshots of the case study, in order to observe changes over a period of time.

Interviews were chosen as the most suitable data collection instrument for observing changes over time. An interview can be performed twice over separate time periods, therefore having the ability to provide 'before' and 'after' sets of data. The data that was 
gained through conducting interviews could also be assessed comparatively using qualitative data analysis methods. As well as being a comparative tool, the data collection method needed to be able to provide qualitative descriptions of key themes that emerged throughout the research. According to Kiddler (1981), face-to-face interviews hold several strengths in qualitative reporting:

- There is a greater capacity to correct misunderstanding by respondents;

- Interviews are better positioned to reveal information that is complex and emotionally laden;

- Interviews can be used to probe the sentiments that may underlie an expressed opinion.

Interviews therefore provided the opportunity to both clarify and further define the themes that arose through the research process.

In terms of the style of the interviews, qualitative research commonly uses the semistructured approach. The semi-structured interview is by definition a set of questions in a set order, which includes many open-ended questions (King, 1994). The semi-structured interview allows for the discovery of the unknown dimension within the case study, and facilitates a greater understanding of what happens in the case and why (Mabry, 2008). The strength of the semi-structured interview is that it can be designed as a theoretically guided approach, while also retaining the flexibility to focus on emergent areas of interest.

In order to conduct longitudinal research, the time period between the data collection sets also needs to be selected (Ruspini, 2002). When assessing changes over time, it is recommended that the interviews are separated by relatively wide spaces of time, such as months or years (Schulsinger et al., 1981). The maximum time span that was allowed between the interviews was seven months. The first set of interviews was conducted in July 2007, and the second set was conducted in March 2008.

\section{Constructing the interview schedule}

There were several factors that shaped the design of the interview. The first was considering how long the interviews were going to be. It was decided that interviews would run for approximately thirty minutes, which meant that each participant would 
undergo two thirty minute interviews over the span of the research. The second consideration was determining the style of the interview questions. Open-ended questions give the interviewee the freedom of expression (Quinn Patton, 2002), and therefore each interview question allowed the participants to freely express their thoughts around each subject area. The final consideration was ensuring that the correct language and wording was used. As highlighted by Fowler (1995), questions involving simple wording avoid ambiguity.

The interview questions themselves were designed to maintain the construct validity of the research. Maintaining construct validity means ensuring that each question is assessing what it is supposed to (De Leeuw, 2008). The purpose of the research was to observe changes on an ingroup and intergroup level within the case study. Mason (2002) states that interviews take a "thematic" and "topic centred" approach in order to ensure that construct validity was maintained. The interview questions were therefore designed in accordance with the relevant literature, to ensure that the questions were gauging the main topics of interest. For example, Question 13 sought to focus on assessing the level of ingroup identification within the case study. The question and the literature that were used to construct the question are shown below:

\section{Interview Schedule - Question \#13}

Topic: Ingroup identification

Literature: "At the individual level, organisational identity represents the distinctive attributes which individuals associate with their membership of a particular organisation." (Empson, 2004, p. 5)

Implications: This shows that member identification is directly linked with the distinctive attributes that a member associates with the group. The interview question would therefore seek to capture the participant's perception of the distinctive nature of the ingroups within the case study as an indicator of ingroup identification.

Question: What is the difference between Blue and Green collections?

Each interview question was constructed in a similar fashion, combining the relevant literature with wording that suited the context of the case study. The full interview schedule is shown Appendix 1. 


\section{Collecting the data}

There are three main steps involved in the data collection process. The steps include identifying the participants, confidentiality and reporting requirements, and conducting and recording the interviews. Each step is explained under the headings that follow.

\section{Sample}

The process of identifying participants began with a formal presentation to all staff within the case study organisation. The presentation, which was conducted by both the senior manger within the organisation and myself, outlined the ingroup development intervention that was going to be implemented within the organisation. There were approximately thirty-five employees at the presentation. Once the presentation was complete, each employee was given an information sheet and consent form which they were to complete if they wanted to take part in the research.

There were initially twenty-four respondents who wanted to participate in the research. All twenty-four respondents were interviewed in the first set of interviews. However, through the seven month duration of the study, four participants either left the organisation or shifted from the case study area to another part of the organisation. The loss of participants is known as the "attrition rate" (Elliott et al., 2008), and having an attrition rate of four meant that only twenty participants could be used in the research. This meant that there were forty research interviews in total: twenty in the first set, and twenty in the second set of data collection. The number of participants and attrition rate are shown in Table 1:

\begin{tabular}{|l|l|l|c|c|c|}
\hline $\begin{array}{l}\text { Consumer Finance } \\
\text { Collections Team }\end{array}$ & $\begin{array}{l}\text { Senior } \\
\text { vs. } \\
\text { Junior } \\
\text { Staff }\end{array}$ & $\begin{array}{l}\mathbf{1}^{\text {st }} \\
\text { Interview } \\
\text { Set }\end{array}$ & $\begin{array}{l}\text { Attrition } \\
\text { Rate }\end{array}$ & $\begin{array}{l}\mathbf{2}^{\text {nd }} \\
\text { Interview } \\
\text { Set }\end{array}$ & $\begin{array}{l}\text { Total \# of } \\
\text { Participants } \\
\text { Used }\end{array}$ \\
\hline \multirow{2}{*}{ Blue Team } & Senior & 3 & 0 & 3 & 7 \\
\cline { 2 - 6 } & Junior & 4 & 0 & 4 & 5 \\
\hline \multirow{2}{*}{ Green Team } & Senior & 5 & 0 & 5 & 2 \\
\cline { 2 - 6 } & Junior & 6 & 2 & 4 & 2 \\
\hline Blue Personal Loans & $\mathrm{n} / \mathrm{a}$ & 3 & 1 & 2 & 2 \\
\hline Green Personal Loans & $\mathrm{n} / \mathrm{a}$ & 3 & 1 & 2 & 20 \\
\hline Total & $\mathrm{n} / \mathrm{a}$ & 24 & 4 & 20 & 2 \\
\hline
\end{tabular}

Table 1: Number of participants and attrition rate 
Confidentiality, ethics, and reporting

There were several mandatory steps that needed to be conducted to ensure that the research could take place. The first step was submitting a research proposal for approval to the Human Ethics Committee of Victoria Management School, Victoria University, Wellington. This was approved on 14 June 2007, and the research began the following month. In addition to this, an information sheet and consent form was distributed to the participants of the research. The information sheet informed potential participants about the details of the study, while the consent form stated that the interviews would remain confidential and that data would be stored at a secure location. These forms are shown in Appendix 2 and Appendix 3 respectively.

\section{Conducting and recording the interviews}

Within the interview itself there were several procedures that took place before the interview questions were asked. Before the interview began the purpose of the interview was discussed with the participants. We also discussed their role as a participant, and my role as a researcher. The participants were asked for consent to tape record the interviews. Permission for this was sought on the consent form that was previously distributed, and participants were asked to bring the consent form with them to the interview. If the request to tape record the interview was declined, the content of the interview would be recorded through note taking. Of the twenty participants that were included in the study, four participants declined the use of a tape recorder.

The interviews were conducted in an organised but relatively informal fashion. This was done so that the construct validity of the research was maintained, while allowing flexibility to further discuss themes that emerged throughout the interview. Maintaining flexibility within the interview was also important for understanding the context of the responses. In many cases, the standardised questions were followed by secondary questions to ensure understanding between both the interviewer and the interviewee.

The system for recording the interviews was a relatively emergent process. For the first set of interviews all recordings were transcribed. Through the process of transcribing the first set of interviews a matrix was designed that was used to record data directly from the 
transcription in order to summarise the interview as a whole. When the second set of interviews were taken the recordings were not transcribed, but instead the data was transferred directly into the matrix. The data that populated the summary matrix was in most cases enough to satisfy the data analysis requirements. However, in the rare case that the matrix was insufficient, the original recordings were referred to for further clarification.

\section{Data analysis}

Content analysis was used to draw themes from the qualitative data that was collected. Within qualitative research, the data collection and data analysis stages go hand in hand (Marshall \& Rossman, 2006), which involves both an inductive and deductive process to identify themes (O'Leary, 2004). For this research, the data analysis process was unique because there were two sets of data collection. This meant that two individual data analysis processes took place, followed by a comparative exercise whereby the individual sets of data were compared and contrasted to look for changes over time. This was done through identifying themes as well as numerically recording commonalities within the data. The following sections give an in-depth description of the analysis process.

\section{Content analysis}

Content analysis is defined as the identifying, coding, and categorisation of patterns found in the data (Patton, 1990). The content analysis process brings forth themes which are unique and differentiated from one another (Cavana et al., 2001). Content analysis can involve several different data analysis methods, even though there may only be one source of data (Creswell \& Plano Clark, 2007). This occurred in the current research, in which a thematic analysis process was conducted, as well as numerically recording commonalities in the data. Another important dimension of the analysis was ensuring that the data was analysed in a "context sensitive" manner (Patton, 1990). Before categorising took place, all responses were considered in relation to the context of the participant as well as the case study as a whole. 


\section{Thematic analysis}

Thematic analysis is concerned with deriving themes from qualitative data. The following steps are adopted from Schutt (2006), and represent the thematic analysis process that took place in this research:

1. Documentation of the data:

- Data analysis began when the first interviews were transcribed. This process involved identifying possible concepts through taking notes and transcribing the data.

2. Organisation/categorisation of the data into concepts:

- This involved reviewing the data in search of commonalities.

- Concepts where consistent in nature, and distinct from other concepts.

- Many of the concepts that were developed through analysing the first interviews were further supported through data from the second interviews.

- New concepts and themes were identified within the second set of interviews.

3. Connection of the data to show how one concept may influence another:

- In some cases, two themes were used to describe the same subject. For example, the theme "Satisfactory Communication" was used in conjunction with the theme "Unsatisfactory Communication". The two themes were designed to give a holistic perspective of the effect that the ingroup intervention had on 'communication'.

4. Corroboration/legitimisation by evaluating alternative explanations, disconfirming evidence, and searching for negative cases:

- The research was able to compare and contrast two stages of data, which allowed the ability to validate or reject themes.

5. Representing the account:

- The themes were named and presented in a way that would exhibit the data accurately. 


\section{Tabulation of data}

As well as subjecting the data to qualitative thematic analysis, commonalities within the data were recorded in table form. Tabulated data, commonly known as "template strategies" (Marshall \& Rossman, 2006), apply sets of codes to the data which are often reviewed and revised throughout the research process. The tabulation process was emergent, and began in the data collection phase when a matrix was designed to capture and summarise the interviews. A set of tables were developed from the interview matrix, and the process worked accordingly:

1. A theme or pattern emerged from the thematic analysis.

2. The reoccurrence of the theme in the first interviews is recorded.

3. The reoccurrence of the theme in the second interviews is recorded.

4. The number of reoccurrences between the first and second set of interviews are compared and contrasted, and linked to the broader context of the analysis.

The tables represented the counting and recording of commonalities found in the data, and therefore served as a valuable tool for verifying the reoccurrence of concepts and themes.

\section{Participant subgroups for data analysis}

The following is a visual representation of the number of participants who were involved in the research: 


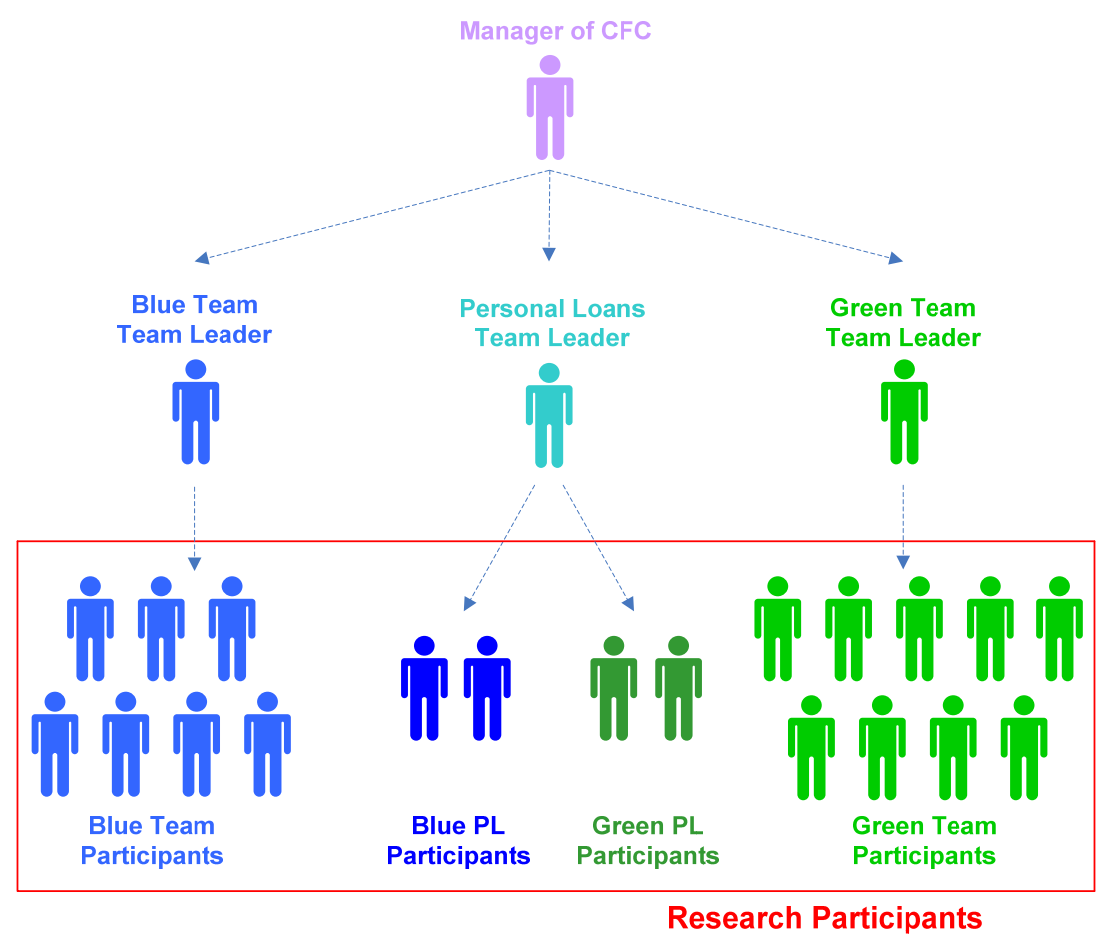

Figure 5: Number and distribution of participants

Participant subgroups were determined by team and experience. As stated in the Case Study Chapter, the Blue and Green Teams are more experienced than the personal loan teams. Within the Blue and Green teams there was a mixture of senior participants and junior participants. It was clear that the senior participants would have a unique perspective in comparison to the junior participants, and it was important to capture this difference in the data analysis. The Blue and Green teams were therefore split into Senior Participants (who had more than eighteen months experience), and Junior Participants (who had less than eighteen months experience). This meant that there were six different participant subgroups used for the research. These are shown in Figure 6 below: 


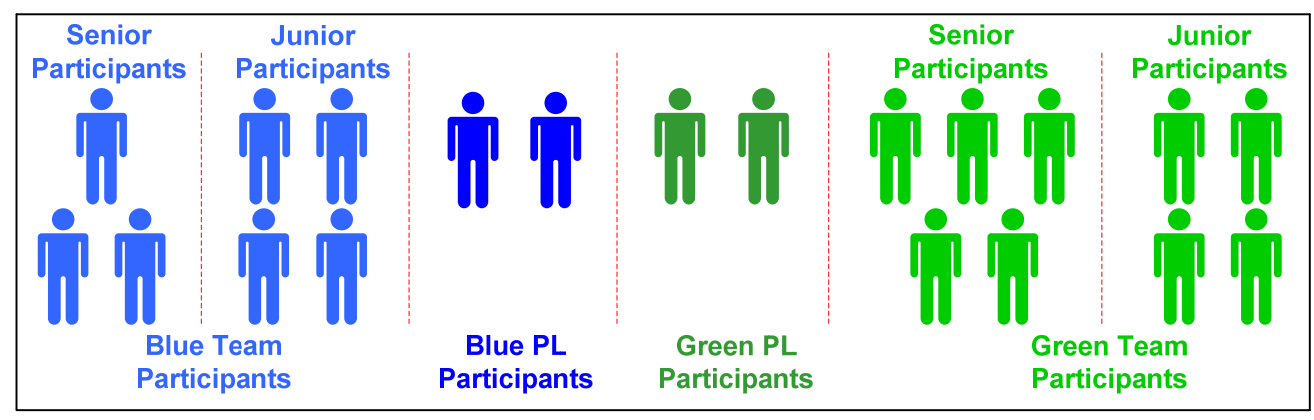

Figure 6: Participant subgroups

Although there are six participant subgroups shown above, there were very few participants from the personal loan teams. Hence, for the purpose of the research the personal loans participants were often analysed as one group.

\section{Conclusion}

The research design aims to assess the effects of the ingroup development intervention. The longitudinal design of the research requires data to be collected at two different points in time. An interview schedule was designed so that it could be used at both the start and the end of the research in order to create a comparative set of data. The steps used to analyse the data are multi-staged, and multi-layered. It involves content analysis using themes and tables, as well as a comparative analysis between the first and second set of interviews. 


\section{Chapter 5}

\section{FINDINGS}

The findings chapter has several sections. The first provides a description of the process which took place in order to implement the ingroup development intervention. This is followed by a summary of the first and second set of interviews. The next section involves identifying the relevant themes that were identified both on an ingroup and intergroup level. The chapter concludes by summarising the themes in table form, and briefly describing the main effects that were observed from the ingroup development intervention.

\section{Implementation: The ingroup development intervention}

The research period spanned from July 2007 to March 2008, and after conducting the first set of interviews the ingroup development intervention was implemented in the case study. The implementation began with seating changes and visual branding of the office environment. Once this was complete, one side of the office belonged to the Blue brand, and the other side of the office belonged to the Green brand. Both the Blue and Green areas were decorated with brand material including posters and other coloured paraphernalia. In addition to the visual branding of the office, each team was educated about their respective brand through the monthly brand meetings. In conjunction with the brand meetings, regular team meetings began on a fortnightly basis. In some cases, the fortnightly team meetings were not maintained due to workload pressures or holiday periods. The final implementation was the posting of team based performance measures. Each team leader had a different approach in how to manage this and there was variance seen in the statistics that were presented, but most performance charts were updated on a daily basis.

In addition to the ingroup development intervention, there were several other events that occurred in the case study during the research period. The first of these events was the introduction of the Recoveries Team to CFC. One month before the second set of interviews, the Recoveries Team began its operations. The previous team leader of the Personal Loan Teams was transferred to be the Team Leader of the Recoveries Team. In 
addition to this, two interview participants form the Green Team were given roles in the Recoveries Team.

The second major event that affected the case study was the onset of the global credit crisis. The credit crisis began in August 2007, one month after the research was initiated. Evidence of the crisis was seen in CFC, as the number of customers falling behind on credit card and personal loan repayments was increasing rapidly. This resulted in an increased workload, and new staff members were hired to manage this situation. The duration of research saw the number of collectors in CFC increase from around thirty-five in July 2007, to approximately fifty in March 2008. A significant number of the new collectors were young employees who were new to Blue Green Bank. The increased workload not only had an impact on the human resources side of the business, but it also affected the performance of CFC. The Green Team had been prepared for the credit crisis, and had hired several new staff before the credit crisis worsened. The team was showing a significant improvement in results. In contrast, the Blue Team was not prepared for the crisis, meaning that the once leading team in CFC slumped to a place where the daily workload was not being completed.

There were several other minor changes which occurred during the research period. The first of these was a "Six Sigma" initiative which focused on the Blue Personal Loans Team. This involved process improvements and several training sessions for the staff. There was also a new CFC bonus system introduced, which established individual performance targets for CFC staff. Before this was implemented, there was no formal bonus or performance structure in place.

\section{Summary of the interviews}

The following section summarises the responses that were observed over each interview set. The first set of interviews that were performed in July 2007 is described first, followed by the second set of interviews that were conducted in March 2008. The summary of each set of interviews is split into three parts according to the participant subgroups. The first part summarises the responses of the Blue Team, the second summarises the responses of the Green Team, and the third gives a joint description of the Personal Loan teams. When 
summarising the responses from a participant subgroup there are three areas of analysis. The first area assesses the responses from the senior participants in the team, followed by the junior participants, and concluding with a summary of the comments in which other teams described the subgroup.

\section{Set 1 interviews}

\section{The Blue Team}

There were a total of seven participants from the Blue Team. The Blue Team included three senior participants and four junior participants. The summary is as follows:

Blue Team - senior participants: The code given to identify senior participants is (Blue SP \#). Comments from the participants described the team as having a "working culture", a term coined by (Blue SP 1). Although the "working culture" was occasionally described in a neutral fashion, it was often explained with negative connotations, highlighting issues such as individualism. One participant described their work colleagues in the Blue Team:

"Some staff work for themselves." (Blue SP 2)

Blue Team - junior participants: The code given to identify junior participants is (Blue JP \#). The junior participants described the nature of the team as being "work focused" (Blue JP 2), and having a "work hard culture" (Blue JP 1), in addition to describing the team as being "performance driven" (Blue JP 1). Some participants made negative comments about the culture in the team:

“...there's no time for anything outside of work." (Blue JP 2)

However, some participants responded positively to this culture:

“...[Blue] card collections set the standard.” (Blue JP 1) 
A key differentiation point between senior and junior participants was the comments on individualism. From the results, it appears that the junior participants did not consciously recognise individualism, and they stated that the team was ultimately cohesive:

“...the [Blue], sort of, side of credit cards collections is, um, like a sort of family feel thing to it, you know. Everyone wants to know how you're doing if you've been away sick, um, or you know, they just ask about how your night was, you know, your evening...” (Blue JP 3)

Although the junior participants did not say that they recognised individualism in the team, the group showed signs of individualism in how they described their approach to work:

"I don't, ah, I don't implement [Blue] type of service, ah, I just do whatever I can do you know, I just do whatever I can...” (Blue JP 4)

Comments about the Blue Team: The following section briefly summarises the statements that other teams made about the Blue Team. The majority of the responses came from the Green Team. The same participant codes are applied to the Green Team: senior participants are (Green SP \#), and junior participants are (Green JP \#).

Green participants described several points of differentiation between themselves and the Blue Team. Green participants generally commented about system differences, work role differences, and the difference in culture between the two teams. Two Green Team participants also highlighted how there were religious differences between the teams:

“...[Blue] has joint religious values.” (Green SP 2)

Green Team participants tended to describe the Blue Team through a competitive lens. Some Green Team participants said that the Blue Team was more advanced in certain areas, such as having better systems (Green SP 2). However, several comments described the Blue Team in a derogative nature. An example of this was one participant describing the Blue Team as being set up like a call centre (Green SP 3). 


\section{The Green Team}

There were five senior and four junior participants from the Green Team. The following is a summary of their responses:

Green Team - senior staff: As previously stated, the code used to identify these participants is (Green SP \#). From the responses it was clear that the senior participants perceived two distinct groups within the Green Team: the older group and the younger group (Green SP 2, 3, 4). However, the responses from the senior participants did not describe the distinction in a negative manner.

The senior participants described 'people' as the strength of their team. This included responses that said that the people in the team were a motivator (Green SP 1), and that the team had a "family feel" (Green SP 3). One participant said that:

"The people, if it wasn't for the people I probably would not be at the bank." (Green SP 4)

The senior participants could also describe the Green Brand. This included descriptions of being more customer focused (Green SP 2), with one participant stating:

“I think, I think we're more, I think we're more tolerant and more understanding, and probably more lenient..." (Green SP 5)

Green Team - junior participants: The junior participants within the Green Team also described the age and experience gap within the team (Green JP 1, 6). The junior participants differed from the senior participants in that they began to articulate that the age/experience gap within the team impacted the overall cohesion of the team. One participant said:

“...the ladies that have been here a lot longer, you know, the older ladies in our team, um, you know, they go out for coffee....and smoke breaks and stuff, and the younger people tend to stick with each other.” (Green JP 2) 
Generally, participants did not elaborate on this division, except for one participant who articulated several negative experiences that they had had with the senior staff in the team (Green JP 1).

Comments about the Green Team: Blue Team participants said that the Green Team had more experience than their own team (Blue SP 2, 3). However, unlike the Green Team who described the Blue Team through a 'competitive lens', the Blue Team described the Green Team through a 'problematic lens'. This is one Blue participant describing the culture of the Green Team:

“...there's a lot of probably, um, things that are pretty much entrenched in [Green] which would probably be, you know, not going to change overnight.” (Blue SP 3)

\section{The personal loan teams}

There were four personal loan participants, including two from the Blue side and two from the Green side. The Blue participants are given the code (Blue PL \#), and the Green participants are given the code (Green PL \#). The following is a summary of the responses from the personal loan participants.

As shown by the comments below, the personal loan teams were new to CFC:

"The team is still very new; therefore it is difficult to tell what the shared values are as they are still being built." (Blue PL 1)

One notion that was beginning to emerge was that of the superiority that the Blue side had over the Green side:

"...the way the team works together...their system (Blue) was already up and working so they've just had it quite easy to operate, and therefore I think they've gelled as a team, and there seems to be a lot more interaction with them." (Green PL 3) 
The following quote is the same participant describing the status of the Green side of personal loans in comparison to the Blue side:

"...management, I think, is focusing on them (the Blue personal loans team) to get them up and running and make sure that they stay operating, because it was already there, um...I feel that...we get noticed and we get helped when we squeal, but if we don't squeal then we just kinda get overlooked." (Green PL 3)

Alternatively, this is a Blue participant describing the Green side of personal loans:

“OK, from what I know, um, [Green] still has, like, paper files so, like, with the [Blue] you just go on the computer, everything's in there..." (Blue PL 1)

Even though the Blue and Green Teams were established at similar times and they had a similar number of staff, at the first set of interviews the Blue side began to emerge as the dominant brand within personal loans.

\section{Set 2 interviews}

The following is a description of the second set of interviews that were conducted in March 2008. These interviews were performed seven months after the first interviews. The results are shown below:

The Blue Team

Blue Team - senior participants: There was an increase in the number of negative comments from senior staff about the state of the Blue Team. One participant described their frustration at the "inconsistency" and "disrespect" of some staff members (Blue SS 3), while another participant described the team as "stressful" (Blue SS 1). An example is shown below:

“...[Blue] does not have much team work." (Blue SP 1) 
In addition to an increase in negative comments, participants exhibited an increased knowledge of the Blue brand. Each participant had a different perspective of the brand, yet participants were confident in articulating what it was:

“...make service a personal relationship.” (Blue SP 3)

Blue Team - junior participants: There were several responses which highlighted the “...strong work culture" (Blue JP 2) within the Blue team. However, what was noticeable was the increase in responses that described positive relationships within the team. Several participants commented on how they saw their colleagues:

“...it's a good place to work...good staff members.” (Blue JP 1)

Other participants described the nature of how the team worked together:

“...it is quite a cohesive unit and we work as a team.” (Blue JP 4)

In contrast to the senior participants, the junior participants made comments which said that they enjoyed the working environment:

“...it's a happy team.” (Blue JP 1)

Comments about the Blue Team: Participants held the view that the Green and Blue sides were inherently different. One participant described their perspective of these differences:

“...I definitely think that there's a huge difference between the people in each of the collection teams." (Green JP 2)

Although the Green participants could describe the differences between themselves and the Blue Team, there was a noticeable reduction in negative descriptions. The following participant was asked how their team differed from the Blue Team:

"I guess the different values...the different groups of people, yeah." (Green SP 4) 
One participant also commented on how the Blue Team had different religious values than the Green Team (Blue SP 4).

\section{The Green Team}

Green Team - senior participants: The senior participants described the age and experience gap within the Green Team. Many of the participants described a positive relationship between the two groups (Green SP 3, 4, 5). One example is given below:

“...previously we have been told we don't work as a team, but we actually did, but now, um, we all, we all get on really well...the newer ones, you know, I think the older ones are prepared to help the newer ones and, and the newer ones are prepared to come to the older ones to ask for assistance..." (Green SP 3)

The senior participants also highlighted that the younger staff have a different way of approaching the role (Green SP 2), and that they often make mistakes:

“...new members are making mistakes that need to be cleaned up." (Green SP 5)

In general, senior participants conveyed that they valued the people in their team and enjoyed being a part of the team:

“...I feel a sense of belonging; being valued.” (Green SP 4)

Participants were also able to describe the Green brand. Participants described the nature of the Green brand as being "customer focused" (Green SP 4), having "good customer service" (Green SP 5), and "good service standards" (Green SP 2).

Green Team - junior participants: Although there was recognition of the age and experience gap within the team (Green JP 1), the junior participants outlined that this was no barrier to the cohesiveness of the team as a whole. One example is shown below:

“....nah, I don't think there's separations in my team, I think we all get along...” (Green JP 6) 
The participants also described how they enjoyed the relationships with their colleagues, and how the 'people' are one of the strengths of the team. One participant said that they are committed to the collections role because of the other people in the team (Green JP 5).

Comments about the Green Team: Comments from the Blue participants indicated that they respected the Green Team. Many of the Blue participants would describe the weakness of their own team in comparison to the Green Team:

“...[Green] staff have more people skills, and are a closer team.” (Blue SP 1)

Blue participants also conveyed that it was more enjoyable to be in the Green Team:

“...[Green] have more fun." (Blue JP 2)

The personal loan teams

Blue participants described their team as "cohesive" (Blue PL 1), and working towards common goals:

“...our team, like, how it motives us, we know if the team's working towards a goal you don't want to let them down, so you're motivated to help them reach the team goals..." (Blue PL 4)

These comments contrasted those of the Green participants, who described the Green side of personal loans as having a "lack of collaboration" (Green PL 3).

Several responses also described the Blue Team as being superior to the Green Team, either in terms of systems or operations. One Green participant describes the Blue personal loans team:

“...they seem to have more interaction." (Green PL 2)

There was also recognition from the Blue participants that their service was superior to the Green side (Blue PL 1). 


\section{Themes from the data}

The longitudinal design of the research allowed for themes to be assessed over time. This meant that data analysis involved not only identifying themes, but tracking changes in the themes over the seven month duration of the research. The themes are presented in two sections. The first section separates the Blue Team and the Green Team from the other collection teams, and highlights themes that were observed in each of these teams. This is followed by the second section which presents themes that were observed over all teams in the case study. This section is further split into two parts, the first describes the themes that relate to ingroup dynamics, and the second outlines themes that discuss intergroup dynamics. A summary of the themes are given below.

\section{Blue Team and Green Team themes}

The following is a description of the themes that relate to either the Blue Team or the Green Team. Due to the fact that these teams were the two largest teams in the case study, a separate analysis was performed to assess the perceived changes within the teams themselves.

\section{The Blue Team}

Theme 1: The team is performance driven

This theme describes a team dynamic that is work focused and motivated by business results. The category originated from Blue Team participants and was further described by responses from the Green Team. The name of the theme was derived from the data when (Blue JP 1) described the Blue Team as "performance driven". The theme was identified in the first interview set, but there were changes observed in the theme over time.

First interviews: Within the first set of interviews there were eight participants who described the team as performance driven. Participants from the Blue Team described this theme in great detail, with some participants describing the theme in a positive manner: 
"...I think the ethics in the team are pretty, are pretty high, as far as work ethics and things like that, yeah.” (Blue SP 3)

However, the majority of the participants saw the performance focus of the team as a negative characteristic:

“...I think that um, um, [Blue] are, are too business driven.” (Blue JP 1)

Second Interviews: There was a significant reduction in the number of participants who described the Blue Team as performance driven. There were four participants who described this characteristic, and these participants were all from the Blue Team. The descriptions in the second interviews were not of a negative nature, as the majority of the participants simply expressed that the team was work focused (Blue SP 2; Blue JP 2).

Theme 2: Positive ingroup relationships

This theme exhibits responses that describe positive relationships within the team. The theme originated from the second set of interviews, where there was a noticeable change in the number of comments which described positive relationships within the team. This is shown below:

First Interviews: There were two participants who described this theme on two separate occasions. These comments related to the "family feel" (Blue JP 3) and the "feeling of togetherness" (Blue JP 2) within the team.

Second Interviews: There were four participants who described this theme on six separate occasions, with one of these participants being from the Green Team. The following participant describes the reasons for his commitment to the organisation:

“...it's the environment, it's the relational, you know...I think more so the people." (Blue JP 1) 


\section{The Green Team}

Theme 1: Separations between experienced and inexperienced staff

This theme relates to comments that describe a separation between the younger (inexperienced) and older (experienced) staff within the team. The theme originated from the first set of interviews as participants described a clear separation between the two groups. The theme was further supported by similar responses within the second set of interviews. The name of the theme originated directly from the data:

“Oh, I think there's separations but I think overall we're, you know, very cohesive, you know, everyone gets on really well and yet the only separation is just age, different ages, but they come together for different things and, yeah." (Green SP 4)

The following is a summary of the theme from each interview set:

First Interviews: In the first interview set there were six participants who contributed to this theme. The theme was viewed differently by each subgroup within the Green Team. The experienced staff generally saw the separation in a positive manner, although the inexperienced participants described a negative relationship between the two groups.

Second Interviews: There were three participants who described this theme. The majority of these responses outlined the difference in age and experience between the two groups, yet the differences were not perceived in a negative manor.

Theme 2: Positive ingroup relationships

A summary of this theme for each interview set is shown below:

First Interviews: The theme included responses which described the team as cohesive, having good team work, and having good relationships:

“...it's working with them, that you get through the day really. You help each other out..." (Green SP 2) 
From the first interviews there were five participants who contributed to this theme, four of which were senior participants.

Second Interviews: There were eight participants who contributed to this theme. What was noticeable was the increase in responses from the junior participants:

“The team gets on really well.” (Green JP 2)

\section{Ingroup and intergroup themes}

The following section describes the themes that were observed over all teams in the case study. This is split into two sections to assess the trends that were observed on an ingroup level, as well those that were observed on an intergroup level. The ingroup themes take data from all participants to depict the general perception of ingroup dynamics, such as observing the cohesiveness of each collection team. In contrast, the intergroup themes describe the trends which are seen on an intergroup level, such as how the different teams relate to each other. The following is a summary of the themes.

\section{Ingroup themes}

Theme 1: Ingroup cohesion

This theme summarises positive ingroup comments. There were a high number of participants who contributed to this theme, and the theme itself was generated in order to assess the ingroups within the case study. This theme describes ingroup cohesiveness within the individual teams, though it does not consider the cohesiveness between the different teams. The following quote represents this theme:

"I'd say it's a fairly cohesive group..." (Green PL 2) 
* The number of participants that contributed to the theme

\begin{tabular}{|l|l|l|}
\hline Team & Set 1 & Set 2 \\
\hline Blue SP & 2 & 2 \\
\hline Blue JP & 4 & 4 \\
\hline Green SP & 3 & 4 \\
\hline Green JP & 2 & 3 \\
\hline Blue PL & 1 & 2 \\
\hline Green PL & 2 & 1 \\
\hline Total \# of Participants who Contributed to the Theme & $\underline{14}$ & $\underline{16}$ \\
\hline
\end{tabular}

Besides the two additional participants who contributed to this theme in the second set of interviews, there were no major shifts observed in this theme over time.

Theme 2: Ingroup separations

This theme describes fractions or disagreements within the separate teams which are either work related or relationship based. Although the theme itself represents an ingroup characteristic, it was a theme which was applicable to the case study as a whole rather than any single team. Although the theme originated from the data, it was designed to accompany the theme, "Ingroup cohesion", for comparative purposes. The theme is represented by the following participant, who was asked what factors would improve their motivation:

"I think, um, honestly...if other staff work a bit harder." (Blue JP 1)

* The number of participants that contributed to the theme

\begin{tabular}{|l|l|l|}
\hline Team & Set 1 & Set 2 \\
\hline Blue SP & 1 & 2 \\
\hline Blue JP & 1 & 1 \\
\hline Green SP & 1 & 1 \\
\hline Green JP & 1 & 0 \\
\hline Blue PL & 0 & 1 \\
\hline Green PL & 0 & 1 \\
\hline Total \# of Participants who Contributed to the Theme & $\underline{4}$ & $\underline{6}$ \\
\hline
\end{tabular}


The number of participants who contributed to this theme increased from four to six.

Theme 3: Satisfactory communication

This theme describes positive responses about the communication system within the case study. The theme emerged from the second interviews, which showed an increase in the number of participants who gave positive descriptions about the communication that they received on a daily basis. The name of the theme was created through the research process, as it appropriately summarised the responses. A representative response is shown below:

"...[communication is] better than what it has been, with regard to keeping us informed of what's going on...at least we know a lot more of where we're going." (Green SP 3)

* The number of participants that contributed to the theme

\begin{tabular}{|l|l|l|}
\hline Team & Set 1 & Set 2 \\
\hline Blue SP & 2 & 1 \\
\hline Blue JP & 2 & 3 \\
\hline Green SP & 0 & 4 \\
\hline Green JP & 1 & 1 \\
\hline Blue PL & 0 & 1 \\
\hline Green PL & 0 & 0 \\
\hline Total \# of Participants who Contributed to the Theme & $\underline{5}$ & $\underline{10}$ \\
\hline
\end{tabular}

The number of positive comments doubled between the first and second set of interviews. This included an increase in the number of participants who said that they had positive dialogue with their manager.

Theme 4: Unsatisfactory Communication

This theme represents negative comments about the communication system within the case study. The theme emerged from the first set of interviews, with a high number of participants giving negative responses about the communication that they received. The 
theme was designed to be compared and contrasted with the theme, "Satisfactory communication". A representative quote is shown below:

“...I guess communication's a little bit slow; it takes a while for things to get back to you at the moment..." (Blue PL 4)

* The number of participants that contributed to the theme

\begin{tabular}{|l|l|l|}
\hline Team & Set 1 & Set 2 \\
\hline Blue SP & 1 & 0 \\
\hline Blue JP & 2 & 0 \\
\hline Green SP & 5 & 1 \\
\hline Green JP & 3 & 2 \\
\hline Blue PL & 2 & 0 \\
\hline Green PL & 1 & 1 \\
\hline Total \# of Participants who Contributed to the Theme & $\underline{14}$ & $\underline{4}$ \\
\hline
\end{tabular}

The majority of comments described a lack of information and feedback. These comments diminished over the duration of the research.

Theme 5: A lack of training and development

This theme expresses a need for more training and development within the case study. The theme originated directly from the first set of interviews. The name of the theme emerged through the research process, because the term "training and development" summarised all comments relating to training, career development, and team development. The following quote represents the theme:

“...we used to actually have weekly meetings, where we could, um, clarify and be cohesive about everybody doing the same thing...we at the moment have so many different people of different ages, different lengths of duration here, um, different training. $\mathrm{OK}$, so we're supposed to be doing the one job, doing it a whole lot different..." (Blue JP 5) 
* The number of participants that contributed to the theme

\begin{tabular}{|l|l|l|}
\hline Team & Set 1 & Set 2 \\
\hline Blue SP & 2 & 1 \\
\hline Blue JP & 4 & 1 \\
\hline Green SP & 3 & 3 \\
\hline Green JP & 2 & 0 \\
\hline Blue PL & 1 & 0 \\
\hline Green PL & 0 & 0 \\
\hline Total \# of Participants who Contributed to the Theme & $\underline{12}$ & $\underline{5}$ \\
\hline
\end{tabular}

There was a significant reduction the number of participants who contributed to this theme.

Theme 6: Participants have brand knowledge

The following theme describes participant awareness of their respective brand. The theme originated from the data, which was prompted by two interview questions. A representative quote came from a participant who was asked to describe the unique characteristics of their brand:

"We try to help customers...we try to help them out...that's my understanding." (Blue JP 4)

* The number of participants that contributed to the theme

\begin{tabular}{|l|l|l|}
\hline Team & Set 1 & Set 2 \\
\hline Blue SP & 2 & 3 \\
\hline Blue JP & 1 & 4 \\
\hline Green SP & 2 & 5 \\
\hline Green JP & 1 & 3 \\
\hline Blue PL & 1 & 1 \\
\hline Green PL & 2 & 2 \\
\hline Total \# of Participants who Contributed to the Theme & $\underline{9}$ & $\underline{18}$ \\
\hline
\end{tabular}

The second set of interviews showed twice as many participants who could describe their brand. 


\section{Intergroup themes}

The following themes are representative of the relationship between the different teams in the case study. The themes are summarised below:

Theme 1: Intergroup cohesion

This theme describes positive relationships between the different teams in the case study. A quote that represents the theme is shown below:

"But, but overall...there seems to be, like, you know, one general focus, like, everybody's aim's for the same kind of things..." (Blue PL 1)

* The number of participants that contributed to the theme

\begin{tabular}{|l|l|l|}
\hline Team & Set 1 & Set 2 \\
\hline Blue SP & 0 & 1 \\
\hline Blue JP & 2 & 0 \\
\hline Green SP & 0 & 2 \\
\hline Green JP & 0 & 2 \\
\hline Blue PL & 0 & 2 \\
\hline Green PL & 0 & 0 \\
\hline Total \# of Participants who Contributed to the Theme & $\underline{2}$ & $\underline{7}$ \\
\hline
\end{tabular}

There was an increase in the number of participants who contributed to this theme. For the first interviews, there were two participants who mentioned intergroup cohesion on two separate occasions. The second interviews saw seven participants mention intergroup cohesion on thirteen separate occasions.

Theme 2: Intergroup separations

This theme represents intergroup bias and discrimination. The theme emerged from the first set of interviews, as there were a high number of participants who described intergroup disharmony. A quote that represents this theme is shown below: 
“...we are supposed to be working as one department...um, it's not happening." (Blue SP 1)

* The number of participants that contributed to the theme

\begin{tabular}{|l|l|l|}
\hline Team & Set 1 & Set 2 \\
\hline Blue SP & 2 & 1 \\
\hline Blue JP & 2 & 0 \\
\hline Green SP & 1 & 0 \\
\hline Green JP & 0 & 0 \\
\hline Blue PL & 0 & 1 \\
\hline Green PL & 2 & 2 \\
\hline Total \# of Participants who Contributed to the Theme & $\underline{7}$ & $\underline{4}$ \\
\hline
\end{tabular}

There was a reduction in the number of participants who contributed to this theme.

Theme 3: No differentiation between teams

This theme represents the inability of the participants to differentiate between the branded teams. The theme includes comments where participants stated that there was no intergroup differentiation within the case study. The purpose of this theme is to show the level of differentiation between the different branded teams. A quote which represents this theme was given by a participant who was asked to describe the difference between the Blue Team and the Green Team:

"We all do the same thing; difference is we finish at eight, they finish at seven." (Blue SP 2)

* The number of participants that contributed to the theme

\begin{tabular}{|l|l|l|}
\hline Team & Set 1 & Set 2 \\
\hline Blue SP & 2 & 1 \\
\hline Blue JP & 1 & 1 \\
\hline Green SP & 2 & 0 \\
\hline Green JP & 3 & 0 \\
\hline Blue PL & 1 & 1 \\
\hline Green PL & 0 & 0 \\
\hline Total \# of Participants who Contributed to the Theme & $\underline{9}$ & $\underline{3}$ \\
\hline
\end{tabular}


There was a significant reduction in the number of participants who contributed to this theme. In the first set of interviews there were nine participants who on sixteen different occasions stated that there was no intergroup differentiation. In the second interviews, only three participants commented on four separate occasions.

Theme 4: Blue teams are superior

The theme describes the perceived superiority of the Blue teams over the Green teams. These comments emerged through both the Blue participants describing themselves, and the Green participants describing the Blue teams. The theme emerged from the first set of interviews. A comment which represents the theme was given by a Blue participant who was describing their own team:

"I think in the [Blue] team, um, I think it sets the benchmark..." (Blue JP 1)

Another representative quote was found when a Green participant was describing the changes that had been occurring in CFC:

“[Blue Green] Bank...they're not so much looking at the best of both, they're not interested in looking at how we worked as [Green], um, from what I've seen and it's not just within our own team, I've heard it from other areas, it's very much 'well this is the way [Blue] did it, this is the way we're going to go"”. (Green SP 3)

* The number of participants that contributed to the theme

\begin{tabular}{|l|l|l|}
\hline Team & Set 1 & Set 2 \\
\hline Blue SP & 0 & 1 \\
\hline Blue JP & 1 & 0 \\
\hline Green SP & 2 & 0 \\
\hline Green JP & 1 & 1 \\
\hline Blue PL & 2 & 1 \\
\hline Green PL & 1 & 1 \\
\hline Total \# of Participants who Contributed to the Theme & $\underline{7}$ & $\underline{4}$ \\
\hline
\end{tabular}


In the first set of interviews, seven participants made ten comments that related to this theme. In the second set of interviews, four participants made four separate comments.

Theme 5: Green teams are resistant

This theme summarises comments which describe the Green teams as resistant. This includes comments from Blue participants who described the Green teams as resistant, as well as some expressions of resistance from Green participants. A representative quote is shown below:

“...[Green] Bank culture, to me, has been totally eroded; I have a lot less job satisfaction doing the job that I'm doing under [Blue], sorry." (Green SP 5)

* The number of participants that contributed to the theme

\begin{tabular}{|l|l|l|}
\hline Team & Set 1 & Set 2 \\
\hline Blue SP & 1 & 0 \\
\hline Blue JP & 2 & 0 \\
\hline Green SP & 4 & 1 \\
\hline Green JP & 0 & 2 \\
\hline Blue PL & 0 & 0 \\
\hline Green PL & 1 & 1 \\
\hline Total \# of Participants who Contributed to the Theme & $\underline{8}$ & $\underline{4}$ \\
\hline
\end{tabular}

There was a reduction in the number of participants who contributed to this theme.

\section{Summary table: Thematic analysis}

The themes that emerged through the data analysis are shown in Table 2: 
Table 2: Thematic analysis summary table

\begin{tabular}{|c|c|c|c|}
\hline Category & Theme Name & $\begin{array}{l}\text { \# of Participant } \\
\text { Contributions } \\
\text { (Set 1/ Set 2) }\end{array}$ & Behaviour Over Time \\
\hline \multirow[t]{2}{*}{ Blue Team } & The team is performance driven & $8 / 4$ & $\begin{array}{l}\text { There was a reduction in this theme, and a reduction in the negative descriptions of the } \\
\text { "performance driven" nature of the Blue Team. }\end{array}$ \\
\hline & Positive ingroup relationships & $2 / 4$ & There was an increase in the number of comments in this area. \\
\hline \multirow[t]{2}{*}{ Green Team } & $\begin{array}{l}\text { Separations between experienced and } \\
\text { inexperienced staff }\end{array}$ & $6 / 3$ & $\begin{array}{l}\text { There was a reduction in the theme over time. The junior participants initially perceived the } \\
\text { "separation" in a negative manner, but this diminished over time. }\end{array}$ \\
\hline & Positive ingroup relationships & $5 / 8$ & $\begin{array}{l}\text { In the first interviews there was only one representative from the junior participants; in the } \\
\text { second interviews there were three. }\end{array}$ \\
\hline \multirow{6}{*}{$\begin{array}{l}\text { Ingroup } \\
\text { Themes }\end{array}$} & Ingroup cohesion & $14 / 16$ & Slight increase in this theme. No major shifts were observed. \\
\hline & Ingroup separations & $4 / 6$ & Increase in this theme. No major shifts were observed. \\
\hline & Satisfactory communication & $5 / 10$ & $\begin{array}{l}\text { Significant increase in this theme over time. Participants more frequently cited positive } \\
\text { dialogue with their manager or team leader. }\end{array}$ \\
\hline & Unsatisfactory communication & $14 / 4$ & Comments stating a lack of information and feedback reduced over time. \\
\hline & A lack of training and development & $12 / 5$ & $\begin{array}{l}\text { A significant reduction was observed, especially in the number of participants who wanted } \\
\text { to be personally developed in their role. }\end{array}$ \\
\hline & Participants have brand knowledge & $9 / 18$ & A significant increase in this theme. \\
\hline \multirow[t]{5}{*}{$\begin{array}{l}\text { Intergroup } \\
\text { Themes }\end{array}$} & Intergroup cohesion & $2 / 7$ & $\begin{array}{l}\text { There was a marked increase in the number of participants and frequency of comments } \\
\text { relating to this theme. }\end{array}$ \\
\hline & Intergroup separations & $7 / 4$ & A reduction in this theme over time. \\
\hline & No differentiation between teams & $9 / 3$ & A reduction in this theme over time. \\
\hline & Blue teams are superior & $7 / 4$ & A reduction in the number of participants and frequency of comments over time. \\
\hline & Green teams are resistant & $8 / 4$ & Reduction in this theme over time. \\
\hline
\end{tabular}




\section{Effects of the ingroup development intervention}

A key part of the research was ensuring that the ingroup development intervention achieved what it set out to do. The overarching purpose of the intervention was to facilitate ingroup identification within the case study. The intervention sought to facilitate ingroup identification by creating intergroup differentiation and increasing ingroup communication. The results suggest that the objectives of the intervention were achieved.

The intervention had a clear impact on ingroup communication within the case study. There were two themes that gauged the level of communication. The first theme was named "Satisfactory communication", which saw an increase from five participants to ten participants. The second theme was "Unsatisfactory communication", which reduced from fourteen participants to four participants. The responses showed that the perception of communication improved over the course of the research. Considering that the ingroup development intervention only included ingroup communication initiatives, the research concludes that the intervention improved the perception of ingroup communication within the case study.

In addition to increased ingroup communication, the intervention had an impact on the perceived level of intergroup differentiation within the case study. There were two key indicators that showed increased differentiation. The first was the increase in the number of participants who could describe their respective brands. In the first interview set there were nine participants who had brand knowledge, whereas in the second set of interviews there where eighteen participants who could describe their respective brand. The second indicator was seen through the theme "No differentiation between teams". There was a reduction from nine participants to three participants, which indicates that after the intervention there was less ambiguity in defining the unique characteristics of each team. Therefore, the ingroup intervention increased the perceived level of intergroup distinctiveness within the case study.

The purpose of developing ingroup communication and intergroup differentiation was to facilitate ingroup identification. The literature states that both increased communication (Empson, 2004) and increased intergroup differentiation (Balmer \& Dinnie, 1999) contribute to identity construction. The literature also defines identification as the extent to 
which the personal characteristics of an individual aligns with the distinctive attributes of the organisation (Hogg \& Terry, 2000). The brand was specific to the ingroups; therefore the increased number of participants who could describe their brand indicates that participants had a greater knowledge of the distinctive attributes of their ingroup. Due to the increase in intergroup differentiation, ingroup communication, and brand knowledge, the research concludes that the intervention developed a greater sense of participant identification with the ingroup.

\section{Key findings}

From the ingroup identification that was generated through the intervention, there were two main effects seen in the case study. The first was a perceived reduction in intergroup status differences between the Blue and Green teams, and the second was an improved perception of both ingroup and intergroup relations. Both effects are described below.

\section{Reduction in the perceived status differences}

The findings suggest that there was a reduction in the perceived status differences between the Blue and Green teams. Within SIT and intergroup research, status differences are seen in the post-merger environment through the high-status group exhibiting superiority, and the low-status group showing resistance. Due to the nature of the acquisition, the Blue teams were seen as superior to the Green teams. The findings showed that there was a reduction in the perceived superiority of the Blue teams. The theme that gauged this was "Blue teams are superior", in which there was a reduction from seven participants to four participants. The findings also showed a reduction in the perceived resistance from the Green teams, as the theme "Green teams are resistant" reduced from eight participants to four participants. The reduction in Blue team superiority and Green team resistance indicates that the ingroup development intervention, and the resulting ingroup identification, prompted a reduction in the status differences between the two brands.

2. Ingroup identification facilitates both ingroup and intergroup relations

The second major finding from the research indicates an improved perception of both ingroup and intergroup relations. In terms of ingroups, the general trend through the 
research was that the participants' views of their ingroup improved overtime. This was seen through a reduction in the number of participants who described the Blue Team as "performance driven", a reduction in the number of participants who believed that there were separations within the Green Team, and an improvement in the perception of ingroup relationships within both the Blue and Green Teams. The only contrasting result was an increase in the number of participants who mentioned separations within the ingroup. However, this was contradicted as there was also an increase in the number of participants who reported ingroup cohesion. The research concludes that overall, the intervention and resulting ingroup identification had a positive impact on ingroup perceptions within the case study.

The data also showed a significant improvement in the perception of intergroup relations. The number of participants who identified intergroup cohesion increased from two to seven, while the number of participants who identified intergroup separations reduced from seven to four. As mentioned previously, the data also exhibited a reduction in the perceived status differences between the branded teams which, as stated by the literature, improves intergroup relations (Haunschild et al., 1994). The findings therefore conclude that facilitating ingroup identification improved the perception of intergroup relations within the case study.

\section{Conclusion}

The chapter summarised the thematic analysis and in turn drew conclusions that can be used for further theoretical discussion. The findings showed that the ingroup intervention facilitated both intergroup differentiation and ingroup communication, which facilitated ingroup identification within the branded teams. The findings state that the ingroup identification that was facilitated through the intervention resulted in a perceived reduction in status differences between the branded teams, as well as an improved perception of ingroup and intergroup relations. 


\section{Chapter 6}

\section{DISCUSSION}

The following section discusses the results of the research in comparison to the literature that surrounds the study. This is described through six main headings which are shown below:

\section{Positive ingroup identification}

The positive effects of ingroup identification have rarely been utilised in the post-merger environment. This is due to the belief that ingroup identification will naturally lead to negative perceptions of the outgroup (Sumner, 1906; Tajfel et al., 1971; Tajfel \& Turner, 1979). Past studies have acknowledged the benefits of a common ingroup identity in the post-merger environment (Gaertner et al., 1996; Van Knippenberg et al., 2002; Van Leeuwen \& Van Knippenberg, 2003; Van Leeuwen et al., 2003). However, the same studies also found that ingroup identification increases intergroup bias and discrimination (Van Leeuwen et al., 2003). The results of this research show that, in a specific context, ingroup identification can improve the perceptions of both ingroup and intergroup relations without causing intergroup bias and discrimination.

The findings were distinctive from the literature through the discovery that ingroup identities were generated without causing a trend of intergroup bias in the responses. This ought to come as no surprise, as past research shows that the majority of perceived ingroup bias is not so much anti-outgroup behaviour as it is pro-ingroup behaviour (Brewer, 1979). The research showed the benefits of pro-ingroup behaviour, a result supported by the literature which states that a common ingroup identity generates positive feelings among group members and improves the cohesiveness of the group itself (Gaertner et al., 1996; Mullen et al., 1992). The research therefore demonstrated the positive effects of ingroup identity within a post-merger environment. 


\section{Ingroup identification reduces the status-differential}

The literature review revealed that ingroup identification can increase the statusdifferential in a merger, which, in turn, inhibits the merger integration process. SIT states that people aspire to be in the high-status group (Tajfel, 1974; Tajfel \& Turner, 1979), and that membership in a low-status group is not sufficient to fulfil this aspiration. If there is a high-status group within a merger, this group can have a feeling of superiority (Marks \& Mirvis, 2001) and can engage in discriminative behaviour to defend their high-status position within the organisation (Seo \& Hill, 2005). In contrast, the low-status group seeks to combat their disadvantaged position through collective action (Tajfel \& Turner, 1979), which is usually seen through "resistance to change" (Donnelly, 1992). Status differences have previously been linked to ingroup identity development, as developing ingroup identity facilitates pre-merger identification which can accentuate historical status differences between the merging organisations (Tajfel et al., 1971; Tajfel \& Turner, 1979). However, in contrast to the literature, the current research shows that instead of increasing the status-differential, the ingroup development intervention reduced the perceived status differences in the post-merger environment.

This research showed that the ingroup development intervention assisted to reduce the perceived status differences between the merged organisations. As shown through the case description, the organisation had already taken steps to reduce the status-differential within the post-merger environment. However, the findings still registered a perceived reduction in status differences after the ingroup development intervention. This was shown as the second set of interviews registered fewer responses that described the high-status group as superior, as well as there being a reduction in responses that described the low-status group as resistant. This effect is rarely seen in the literature, as SIT and intergroup studies tend to see ingroup identification as a generator of intergroup differences, which ultimately leads to bias and discrimination (Tajfel et al., 1971; Tajfel \& Turner, 1979). The findings are therefore unique in showing that ingroup identity development participated in a perceived reduction in the post-merger status-differential. 


\section{Low status-differential allows for ingroup identity development}

The literature states that specific environmental factors are required in order to realise the positive effects of generating ingroup identification. Ingroup identification can generate positive effects such as cohesiveness between ingroup members (Gaertner et al., 1996), but this can be turned into anti-outgroup behaviours if there is a status-differential between the merging groups (Haunschild et al., 1994). This research demonstrated only the positive effects of ingroup identification, indicating that there was a low status-differential within the case study environment. Therefore, understanding the factors that formed the case study environment is important for knowing what context enables the positive use of ingroup identification.

The merger integration strategy used by the case organisation formed a context that was conducive for developing ingroup identification. The integration strategy was a "merger of equals" which levelled the degree of pre-merger identity within the post-merger environment (Giessner et al., 2006). Both Blue Bank and Green Bank essentially had an equal degree of power, which reduced status differences and diminished the possibilities for intergroup bias and discrimination (Tajfel et al., 1971; Tajfel \& Turner, 1979). The organisation also pursued a "third culture" approach (Cartwright \& Cooper, 1993), whereby none of the pre-merger brands were emphasised in the post-merger environment, opting instead for a neutral integration strategy. The organisation also promoted intergroup contact through initiating joint-brand, multi-departmental performance meetings, which according to the literature eliminates intergroup misperceptions and allows for the realisation of intergroup commonalities (Allport, 1954; Brown, 2000; Green, 2008). The integration strategy that was used by the organisation resulted in a post-merger environment with a low status-differential between the merged groups.

The formation of the low status-differential within the case study was assisted by the time lapse between the merger and the research itself. Although the literature states that postmerger integration can last up to seven years (Stybel, 1986), the four-year gap between the merger and the research clearly alleviated some of the commonly observed post-merger difficulties. The time lapse also shaped the demographic of the staff within the case study. Only four out of the twenty participants involved in the research were employed within the case study before the Blue Bank acquired the Green Bank in 2003. This meant that, for the 
majority of participants, the ingroup intervention was not an exercise in rejuvenating premerger identity and culture, as much as it was a case of organisational identity construction.

\section{Reduced pre-merger identity results in low organisational identification}

The findings showed that the research participants positively responded to organisational identification, rather than the restoration of pre-merger identities. Pre-merger identity refers to the identity that pre-merger groups bring to a merger (Datta et al., 1990; Graves, 1981; Olie, 1994), whereas organisational identity refers to the identity of an organisation as a whole (Seo \& Hill, 2005). The majority of research participants were employed well after the merger took place, which meant that there was no restoration of pre-merger identities for these participants, but rather there was education about their current organisational identity. The ingroup development intervention differentiated and communicated the centrality of their brand, an activity which is defined as organisational identity construction (Empson, 2004). The positive response to organisational identity construction indicates that prior to the intervention, participants had a low level of organisational identification.

Organisational identification is commonly linked to the distinctiveness of the organisation (Albert \& Whetten, 1985; Balmer \& Dinnie, 1999; Empson, 2004; Hogg \& Terry, 2000). Albert and Whetten (1985) describe the identity of an organisation as its central, distinctive, and enduring characteristics. Hogg and Terry (2000) state that an individual's identification with an organisation is their personal alignment with these distinctive attributes, resulting in self-definition according to the organisation of which he or she is a member (Mael \& Ashforth 1992). The centrality and distinctiveness of the organisation can been seen through activities such as branding (Gylling \& Lindberg-Repo, 2006), corporate communications (Balmer \& Dinnie, 1999), and image management (Dutton et al., 1994; Melewar \& Jenkins, 2002; Olins, 1995). Before the ingroup development initiative was implemented, none of the listed mediums for organisational distinctiveness were evident within the case study.

Prior to the ingroup development initiative, the case study environment did not externalise a clear organisational identity. This was exhibited through the large number of participants 
who, from the first interviews, showed a lack of brand knowledge. Of the twenty participants in the research, only nine could describe the brand that they worked for. The cause of this could be assigned to the lack of brand communication, but also the fact that there was no visual communication in the case study environment. The case analysis described an office environment which had no visual representation of either the corporate or the individual brands. The literature would suggest that the lack of distinctiveness within the organisational environment reduced the levels of organisational identification within its members.

The lack of organisational identity within the case study can also be linked to the merger strategies that were used in the case study. Each merger strategy was used to reduce premerger identity in order to establish a new culture in the merged organisation. As shown through the case analysis, the new culture was not diligently supported, and the result was an organisational environment which did not demonstrate a distinctive character. The research links this effect with a lack of organisational identification among the case study participants. Inadvertently, the lack of organisational identity within the case study formed an environment that was conducive to ingroup identity development, as participants appeared to benefit from learning about the current brand of which they were a now a member.

\section{Reduced organisational identification allows for the effective use of ingroup initiatives in the post-merger environment}

The low status-differential and low levels of organisational identification made the case study environment conducive for the effective use of ingroup development initiatives. The ingroup development intervention that was used for the research involved several initiatives which are acknowledged in the literature. The literature states that increased dialogue can promote shared vision, trust in leadership, and increased motivation (Pincus et al., 1991). The literature also states that communicating the goals of the company to the employees will increase productivity and morale (Eisenberg \& Witten, 1987). Communication can also be used through manipulating the physical design of the office to represent certain core values, thus showing the identity of the organisation (Melewar et al., 2006). Visual communication is also seen in branding activities which connect the employees with the organisation (Basu, 2006). All of the above actions were implemented 
on an ingroup level within the case study which, due to the previously mentioned factors, resulted in positive ingroup and intergroup results. The findings therefore suggest that within a specified environment, ingroup development initiatives can be a valid part of postmerger integration strategies.

\section{Intergroup differentiation in the post-merger joint-brand environment}

The literature states that intergroup differentiation is detrimental to post merger-integration (Tajfel et al., 1971; Tajfel \& Turner, 1979), but the current research suggests that this theory may not apply in joint-brand mergers. A merger accentuates pre-merger organisational membership which increases intergroup differences. In most mergers, this is said to hinder the merger process (Mullen et al., 1992). In response to this, merger strategies seek to re-categorise two merging organisations into one new group, which involves eliminating intergroup differences and establishing intergroup commonalities (Van Knippenberg et al., 2002). However, eliminating intergroup differences is difficult in a joint-brand merger, as both pre-merger brands are maintained in the post-merger environment (Basu, 2006). The existence of two brands means the existence of two different identities (Balmer, 1998; Nandan, 2005; Pruzan, 2001), which suggests that intergroup distinctiveness may be required in a joint-brand merger.

As shown by Basu (2006), a joint-brand environment involves the continuation of both pre-merger brands. The literature states that branding activity is the proliferation of the identity of the organisation through the communication of core values (Dutton et al., 1994; Gylling \& Lindberg-Repo, 2006; Olins, 1995). Therefore, having two brands means that two identities are communicated to organisational members. The literature states that in order to generate positive organisational identification, the character of the organisation must be expressed (Albert \& Whetten, 1985; Hogg \& Terry, 2000) and constantly communicated (Empson, 2004). Because of this, eliminating intergroup distinctiveness is difficult within a joint-brand merger due to the fact that the identity of each brand is being communicated, and must be expressed in order to generate positive identification within its members. This research sought to explore the effects of enhancing both post-merger brands. 
The ingroup development intervention created intergroup differentiation by constantly communicating the distinctiveness of each post-merger brand. The research findings showed that post-intervention the branded teams were perceived as more distinctive, which facilitated positive organisational identification and improved ingroup and intergroup perceptions. The findings of the research therefore suggest that in a post-merger environment which maintains two separate brands, intergroup distinctiveness plays an important role in identification, and improves the resulting integration process.

\section{Conclusion}

The research showed that ingroup identification plays an important role within specified organisational contexts. Prior to the ingroup development intervention, the case was one which had experienced low levels of status and organisational identification. The intervention facilitated ingroup identification resulting in a perceived reduction in the status-differential, increased organisational identification, and positive ingroup and intergroup relations. 


\section{Chapter 7}

\section{CONCLUSION}

This final chapter covers four main areas. The first section revisits the research question, and then explains how this was answered through the study. The second section addresses the implications of the research, and then outlines the factors that limited the study as a whole. The final section describes areas for future research.

\section{Research question}

The question that guided the research was: What are the effects of ingroup development in a post-merger joint-brand context? The research question was answered by studying an ingroup development intervention that was initiated in Blue Green Bank. The ingroup development intervention was assessed by performing forty semi-structured interviews through two sets of data collection that spanned a period of seven months. The following points summarise the effects that were seen through the intervention:

1. A reported increase in ingroup communication and intergroup differentiation;

2. The facilitation of ingroup identification;

3. A perceived reduction in the status-differential between the merged groups;

4. An improved perception of both ingroup and intergroup relations.

There were several major shifts that were identified on an ingroup and intergroup level within the case study. For the ingroups in the case study, the results showed an increase in positive communication ratings. Another increase was seen in the number of participants who could articulate their respective brand. In addition to this, there was also a reduction in the demand for training and development. On an intergroup level, there was a marked increase in the number of participants who described cohesion between the different branded teams, and a reduction in the number of participants who identified separations between the different teams. There was also a reduction in the number of participants who described the Blue teams as superior, and fewer participants who described the Green teams as resistant. The theme throughout the findings was an improved perception of 
ingroup and intergroup relations, and a reduced status-differential between the Blue and Green brands within the case study.

Perceptions of the Blue Team improved over the course of the research. In the first interviews, participants described the Blue Team as "performance driven". This perception eroded over time, and there was a reduction in the number of comments from Blue Team participants who described the performance culture in a negative way. There was also an observed increase in the number of participants who identified positive relationships within the team. However, the senior participants of the Blue Team began to increasingly comment on the negative characteristics of the team as a whole. The majority of these comments were directed towards new staff, and these complaints aligned with the increasing work pressure that the Blue Team was facing at the time of the second interviews. This finding contrasted other data which reported an improved perception of ingroup relations both in the Blue Team and in CFC as a whole. Therefore, this result is not considered to be a representation of the overall findings. In summary, there was an observed improvement in the way participants described the Blue Team.

The Green Team showed improvement in how members perceived each other and related to the team as a whole. The first interviews illustrated that participants saw a clear demarcation between the senior and junior staff within the team. Some of the junior participants described the demarcation as having a negative effect. In the second set of interviews, the number of negative comments about the relationship between the senior and junior staff reduced, and there was a noticeable improvement in the way in which the junior staff described the team. This relays an improvement in the cohesiveness of the group as a whole, an effect that could have been facilitated by the ingroup development intervention.

Through analysing the findings and assessing the separate teams, it was clear that the unique characteristics of the case study were a prime determinant of the results. There were two main characteristics that defined the case study, the first being a low statusdifferential, and the second was a low level of organisational identification. The research suggests that these factors create a context that is suited to ingroup initiatives, and that the use of ingroup initiatives in this environment can produce positive organisational identification. The research also suggests that intergroup distinctiveness is a key dimension 
of the post-merger joint-brand environment, as a lack of distinctiveness could result in low levels of identification. The unique characteristics of the research context were derived largely from the case study being a post-merger joint-brand organisation.

The complexity of the post-merger joint-brand organisation was a contributing factor in the findings of the research. Before the ingroup development intervention, there was little brand education or communication within the organisation, and the case analysis described an environment which had a low level of intergroup differentiation. The findings linked the low levels of intergroup differentiation with low levels of organisational identification. The lack of brand communication as well as the inflow of new staff with no historical brand affiliation produced a context that was relatively free from the status-differentials that are commonly generated in M\&As. This made the case study conducive to ingroup identity development, as the findings suggest that the ingroup intervention generated positive organisaitonal identification. This had a positive effect on both ingroup relations and the intergroup dynamics of the case study.

The following model summarises the key phases of the research: 


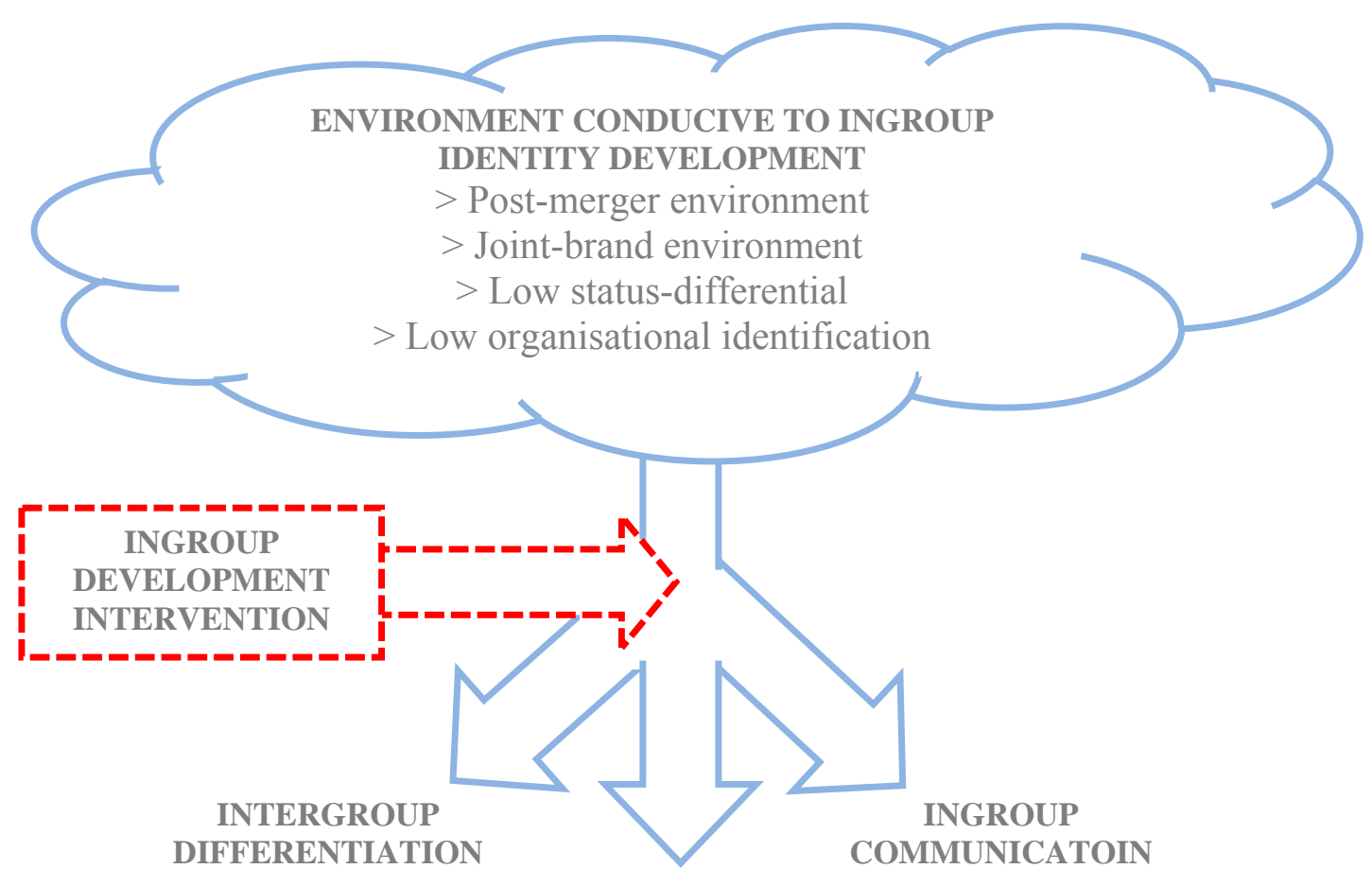

INGROUP

IDENTIFICATION

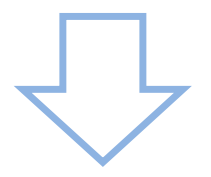

KEY FINDINGS

* The ingroup development intervention reduced the perceived intergroup status-differential.

* Increased ingroup identification has the potential to facilitate positive ingroup and intergroup relations.

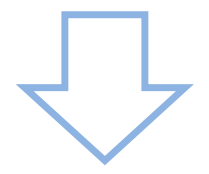

\section{THEORETICAL CONSIDERATIONS}

1. Positive Ingroup Identification.

2. Ingroup identification reduces the status-differential.

3. Low status-differential allows for ingroup identity development.

4. Reduced pre-merger identity results in low organisational identification.

5. Reduced organisational identification allows for the effective use of ingroup initiatives in the post-merger environment.

6. Intergroup differentiation in the post-merger joint-brand environment

Figure 7: Summary of the research 
In summary, the ingroup development intervention generated intergroup differentiation and ingroup communication within the case environment, which then facilitated ingroup identification. This had two main effects, the first being a perceived reduction in the intergroup status-differential, and the second being an improved perception of ingroup and intergroup relations. As shown in the Figure 7, these results were enabled by the set of environmental factors which were unique to the case itself. The findings of the research have implications for both the SIT and intergroup foundations of the research, and also for practitioners in the field of post-merger integration.

\section{Implications for the literature}

The findings of the research hold significance for the theoretical foundations of the study. The inquiry was based on an SIT perspective of the post-merger environment, focussing on the ingroup and intergroup relations within a joint-brand context. The ingroup development intervention showed an improved perception of status and intergroup dynamics, a result which differs from the current literature. These findings hold significance for two main areas of research, which are SIT and intergroup relations, and post-merger integration.

The literature suggests that merger integration is hindered because of the pre-merger identity and culture that each organisation brings to a merger (Datta et al., 1990; Graves, 1981; Olie, 1994). The literature also suggests that many of the people-related problems that are exhibited within a merger are associated with pre-merger identity and culture (Graves, 1981; Hogg \& Terry, 2000; Marks \& Mirvis, 2001; Zaheer et al., 2003). Within a post-merger joint-brand environment, the individual identities relate to branded ingroups, and the literature states that identification with the ingroup can cause anti-outgroup orientations (Tajfel et al., 1971; Tajfel \& Turner, 1979). Pre-merger identity and culture are therefore considered to be a barrier to merger integration, and the literature recommends that both elements are reduced in the post-merger environment (Buono \& Bowditch, 1989; Cartwright \& Cooper, 1993; Gaertner et al., 1990; Giessner et al., 2006). For this reason, the literature does not promote the development of ingroup identification as it can generate the negative effects which are seen through pre-merger identity and culture.

The findings of the research differed from the literature in that ingroup identification and intergroup differentiation were shown to have a positive effect on the intergroup dynamics 
of the case study. The research findings showed that the ingroup intervention improved the perception of intergroup relations, an effect that is rarely seen in intergroup studies. Although the context of the case study played a significant role in the results, the findings are considerable as they question the foundational belief that ingroup identification leads to intergroup bias and discrimination. In conjunction with other ingroup studies (Brewer, 1979; Haunschild et al., 1994), the findings suggest that ingroup identification is only detrimental to intergroup relations in cases of unequal status. This research also concurs with other ingroup literature in stating that ingroup identification has a positive effect on ingroup relations. However, this study goes one step further and opens the possibility for ingroup identification to be a positive force on intergroup relations. This makes ingroup identification a valid consideration for the management of intergroup relations, which balances the typically unaccompanied intergroup approach that is commonly adopted in intergroup studies. The findings therefore unlock another dimension of the complexity of intergroup relations.

The research showed that intergroup cohesion is not only a process of generating intergroup commonalities, but is a complex equation involving considerations of ingroup dynamics and member identification. The increased focus on ingroups essentially draws to the heart of SIT, which considers identification to be a dynamic that exists between the individual and the group to which they are a member (Seo \& Hill, 2005). Identity studies state that a group has a series of central, distinct, and enduring characteristics (Albert \& Whetten, 1985), and that identification with the group is achieved when members align to these distinct characteristics (Mael \& Ashforth, 1992). The current research increased the distinctiveness of the ingroups to facilitate ingroup identification, which in turn had a positive effect on ingroup and intergroup perceptions. Intergroup studies would typically view this action as the increase of intergroup differentiation, which would ultimately lead to intergroup bias and discrimination (Tajfel et al., 1971; Tajfel \& Turner, 1979). However, the literature doesn't adequately consider the forces of identification, which in the current study, showed to be a key factor which caused positive ingroup and intergroup relations. For intergroup studies, this widens the considerations of intergroup relations to include issues of ingroup identification.

The discussion of identification brings into consideration the claim from the literature that intergroup differentiation is a cause of intergroup bias and discrimination (Tajfel, et al., 
1971; H Tajfel \& Turner, 1979). SIT literature states that merger activity accentuates premerger organisational membership (Mullen et al., 1992), which in turn increases intergroup differences, and consequently has a negative effect on intergroup relations (Mullen et al., 1992). A merger is commonly seen as the re-categorisation of two different social groups into one new group (Van Knippenberg et al., 2002), and therefore integration strategies seek to reduce intergroup differences in favour of encouraging a single organisational identity (Buono \& Bowditch, 1989; Cartwright \& Cooper, 1993). In light of this, the literature recommends merger strategies that look to unify ingroups through a common intergroup identity, whereas the findings of the research suggest that a different approach may apply in the joint-brand organisation.

The findings showed that within the joint-brand environment, intergroup differentiation helped facilitate ingroup identification, which, in turn, improved the perceptions of ingroup and intergroup relations. These findings have implications for intergroup research by providing evidence that intergroup differentiation may not necessarily be a prime cause of intergroup bias and discrimination. This research represents a case in which an increase in intergroup differentiation improved the perceptions of intergroup relations, showing that there was no causal link between intergroup differentiation and intergroup bias and discrimination. The possibility that intergroup differentiation is not a prime cause of intergroup difficulties broadens the possibilities for intergroup relations, allowing for the consideration of ingroup distinctiveness in the study of intergroup relations.

\section{Implications for practitioners}

The findings of the research hold several implications for managers who operate in a postmerger joint-brand organisation. On a foundational level, the positive results that were seen through ingroup identification highlight the potential for continuing pre-merger ingroup identities. The literature states the importance and benefits of organisational identification (Ashforth \& Mael, 1989; Dutton et al., 1994), and findings from the current research suggest that ingroup identities play a role in this area of study. Prior to the ingroup development intervention being implemented in the case study, the identity of the branded teams were not emphasised since a "no brand" approach had been taken. However, the findings showed that building the individual ingroups through increased ingroup communication and differentiation had a positive impact on the degree to which 
participants identified with the organisation. Therefore, the continuation of pre-merger ingroup identity and culture are a consideration for managers operating in a post-merger joint-brand environment.

In accompanying the continuation of ingroup identities, the findings showed that in a low status environment the use of intergroup distinctiveness and ingroup communication are potentially effective tools. The findings showed the need for managers to consider the identification of individual teams, which for the current research entailed a series of ingroup initiatives. The ingroup initiatives differentiated the branded teams through branding activities, and facilitated greater degrees of ingroup communication through initiating team meetings and performance updates. The literature states that identification is facilitated through exhibiting the distinctiveness of the brands (Hogg \& Terry, 2000) and frequently communicating this to staff (Empson, 2004), which for this research resulted in positive ingroup and intergroup perceptions. The findings therefore identify the potential in positively differentiating teams within an intergroup context.

\section{Limitations of the research}

There were several factors that limited the study, which ranged from the parameters of the research to the methodology which was used to guide the inquiry. The following section summarises these limitations, and shows the steps which were taken to reduce some of these effects. Simultaneously, the study recognises that its findings are contextually bound, and these findings mainly offer an in-depth understanding of the potential for SIT to contribute to the areas of post-merger integration and intergroup relations.

The first limiting factor was that the research was confined to a single case study. This factor limited both the size and scope of the inquiry. Although single case designs generally provide more detailed descriptions (Schulsinger et al., 1981; Stake, 2005), expanding the number of cases would have made the findings applicable to a wider audience. Because of this, the findings are accompanied by a clear definition of the context of the case study to ensure that the results are interpreted appropriately.

When assessing the limitations of the methodology, the strengths of the research were also potential weaknesses. While the longitudinal design provided vital comparative data for the 
research, it was somewhat hampered by the 'attrition rate' of participants, whereby the number of participants in the research reduced from twenty-four to twenty due to unforeseen withdrawals. The attrition rate of four participants meant that the research was less able to give an accurate representation of the effects of the ingroup development intervention.

The use of only one data collection instrument also posed some limitations for the research. Having only one data collection instrument raises questions over the internal validity of the research. Internal validity is the ability to justify causal relationships that are identified within the data (Kidder, 1981). The research did, however, have several strengths which supported the validity of the study. The first of these strengths was the longitudinal design, whereby two interview sets were taken. Having two sets of data allowed for comparative analysis and the validation of themes. Another key strength was the way in which the data was analysed, as the qualitative measures included thematic analysis which were summarised in table form. The tabulated results provided a summary of the qualitative analysis and gave a holistic perspective of the results. This proved to be a trustworthy tool which was used to validate or reject themes that were proposed throughout the analysis. Finally, understanding the context of the case study helped to accurately interpret the data. A detailed case description provided the context for the responses, which helped to identify authentic themes from the data.

\section{Future research}

The research identified the potential for further study in the area of ingroup identification. The findings question the premise that ingroup identification generates intergroup bias and discrimination. Although the findings suggest a positive relationship between ingroup identification and intergroup relations, the research considered only one organisational context which was tempered by a low status-differential and a low level of organisational identification. The relationship between ingroup identification and intergroup relations needs to be explored in other intergroup contexts in order to broaden the application of the findings. Facilitating ingroup identification in different intergroup environments would build understanding of the limits to which ingroup identification can be used to positive effect. 
The results of the research showed the validity of intergroup differentiation within an intergroup context. This questions the premise that intergroup differentiation is a prime cause of intergroup bias and discrimination, and shows that further research is needed to gain a better understanding of the effects of intergroup differentiation within specified contexts. Intergroup differentiation could also be explored under the lens of identification, since the research suggests that the improved perceptions of ingroup and intergroup relations were exhibited because of the positive identification that was created from the ingroup development intervention. 


\section{REFERENCES}

Albert, S., \& Whetten, D. A. (1985). Organizational Identity. Research in Organizational Behaviour, 7, 263-295.

Allport, G. W. (1954). The Nature of Prejudice. Reading: Addison-Wesley.

Alvesson, M. (2002). Understanding Organizational Culture. London: Sage Publications.

Alvesson, M., \& Wilmott, H. (2002). Identity Regulation as Organizational Control: Producing the Appropriate Individual. Journal of Management Studies, 39(5), 619644.

Ashforth, B. E. (1985). Climate Formation: Issues and Extensions. Academy of Management Review, 10(4), 837-847.

Ashforth, B. E., \& Mael, F. (1989). Social Identity and the Organization. Academy of Management Review, 14(1), 20-39.

Balmer, J. M. T. (1998). Corporate Identity and the Advent of Corporate Marketing. Journal of Marketing Management, 4, 963-996.

Balmer, J. M. T., \& Dinnie, K. (1999). Corporate Identity and Corporate Communications: The Antidote to Merger Madness. Corporate Communications, 4(4), 182.

Basu, K. (2006). Merging Brands after Mergers. California Management Review, 48(4), 28.

Benson, J., \& Foley, J. (2005). When Banks Merge, What Happens to the Brand?

Retrieved 2 October 2008, from

http://www.brandchannel.com/papers review.asp?sp id=620.

Birkinshaw, J., Bresman, H., \& Hakanson, L. (2000). Managing the Post-Acquisition Integration Process: How the Human Integration and Task Integration Processes Interact to Foster Value Creation. Journal of Management Studies, 37(3), 395-425.

Brewer, M. B. (1979). Ingroup Bias in the Minimal Intergroup Situation: A Cognitive Motivational Analysis. Psychology Bulletin, 86, 307-324.

Brown, (2000). Social Identity Theory: Past Achievements, Current Problems, and Future Challenges. European Journal of Social Psychology, 30(6), 745-778.

Buono, A. F., \& Bowditch, J. L. (1989). The Human Side of Mergers and Acquisitions. San Francisco.

Cartwright, S., \& Cooper, C. (1993). The Role of Culture Compatibility in Successful Organisational Marriage. Academy of Management Executive, 7(2), 57-70. 
Cartwright, S., \& Cooper, C. (1996). Managing Mergers Acquisitions and Strategic Alliances: Integrating People and Cultures. Butterworth-Heinemann.

Cavana, R. Y., Delahaye, B. L., \& Sekaran, U. (2001). Applied Business Research: Qualitative and Quantitative Methods. John Wiley \& Sons, Inc.

Covin, T. J., Sightler, K. W., Kolenko, T. A., \& Tudor, R. K. (1996). An Investigation of Post-Acquisition Satisfaction with the Merger. Applied Behavioural Science, 32(2), $125-142$.

Creswell, J. W., \& Plano Clark, V. L. (2007). Designing and Conducting Mixed Methods Research. Thousand Oaks: Sage Publications.

Datta, D. K. (1991). Organisational Fit and Acquisition Performance: Effects of PostAcquisition Integration. Strategic Management Journal, 12(4), 281-297.

Datta, D. K., Pinches, G. E., \& Narayanan, V. K. (1990). Factors Influencing Wealth Creation from Mergers and Acquisitions: A Meta-Analysis. Strategic Management Journal, 13(1), 67 - 84.

De Chernatony, L. (2006). From Brand Vision to Brand Evaluation: The Strategic Process of Growing and Strengthening Brands: Butterworth-Heinemann.

De Leeuw, E. (2008). Self-Administered Questionnaires and Standardized Interviews. In P. Alasuutari, L. Bickman \& J. Brannen (Eds.), The Sage Handbook of Social Research Methods. Trowbridge: Sage Publications.

Denzin, N. K., \& Lincoln, Y. S. (1994). Introduction: The Discipline and Practice of Qualitative Research. In N. K. Denzin \& Y. S. Lincoln (Eds.), Handbook of Qualitative Research (pp. 1-32). London: Sage Publications.

Dinnie, K. (2005). The Pirate Inside: Building a Challenger Brand Culture within Yourself and Your Organization. Journal of Brand Management, 12(4), 305.

Doise, W. (1978). Groups and Individuals: Explanation in Social Psychology. Cambridge: Cambridge University Press.

Donnelly, J. H. (1992). Managing Mergers and Other Changes. Bank Marketing, 24(9), 38.

Drucker, P. F. (1981). The Five Rules of Successful Acquisition. Wall Street Journal, A28.

Dutton, J. E., Dukerich, J. M., \& Harquail, C. V. (1994). Organizational Images and Member Identification. Administrative Science Quarterly, 39(34), 239-263.

Eisenberg, E. M., \& Witten, M. G. (1987). Reconsidering Openness in Organizational Communication. Academy of Management Review, 12(3), 418-426. 
Elliott, J., Holland, J., \& Thomson, R. (2008). Longitudinal and Panel Studies. In P. Alasuutari, L. Bickman \& J. Brannen (Eds.), The Sage Handbook of Social Research Methods. Trowbridge: Sage Publications.

Empson, L. (2004). Organizational Identity Change: Managerial Regulation and Member Identification in an Accounting Firm Acquisition. Accounting, Organizations and Society, 29(8), 759-781.

Fridolfsson, S., \& Stennek, J. (2005). Why Mergers Reduce Profits and Rise Share Prices A Theory of Pre-Emptive Mergers. Journal of the European Economic Association, 3(5), 1083-1104.

Fulmer, R. M., \& Gilkey, R. (1988). Blending Corporate Families: Management and Organization Development in a Post-Merger Environment. Academy of Management Executive, 2(4), 275-283.

Gaertner, S., Mann, J., Dovidio, J., Murrell, A., \& Pomare, M. (1990). How Does Cooperation Reduce Intergroup Bias. Journal of Personality and Social Psychology, 59(4).

Gaertner, S. L., \& Dovidio, J. F. (2000). Reducing Intergroup Bias: The Common Ingroup Identity Model. Miami: Taylor \& Francis.

Gaertner, S. L., Dovidio, J. F., \& Bachman, B. A. (1996). Revisiting the Contact Hypothesis: The Induction of a Common Ingroup Identity. International Journal of Intercultural Relations, 20(3/4), 271-290.

Gage, N. L. (1989). The Paradigm Wars and their Aftermath: A "Historical" Sketch of Research on Teaching Since 1989. Educational Researcher, 18(4), 4-10.

Gerring, J. (2007). Case Study Research: Principles and Practices. New York: Cambridge University Press.

Giessner, S. R., Tendayi, V. G., Otten, S., Terry, D. J., \& Tauber, S. (2006). The Challenge of Merging: Merger Patterns, Premerger Status, and Merger Support. Personality and Social Psychology Bulletin, 32(3), 339-352.

Graves, S. C. (1981). A Review of Production Scheduling. Operations Research, 29(4), 646-675.

Green, T. (2008). Discomfort at Work: Workplace Assimilation Demands, Social Equality, and the Contact Hypothesis. North Carolina Law Review, 86, 379.

Greenwood, R., Hinings, C. R., \& Brown, J. (1994). Merging Professional Service Firms. Organizational Science, 5, 239-257. 
Guba, E. G. (1990). The Alternative Paradigm Dialog. In E. G. Guba (Ed.), The Paradigm Dialog (pp. 17-27). Newbury Park: Sage Publications.

Gylling, C., \& Lindberg-Repo, K. (2006). Investigating the Links Between a Corporate Brand and a Customer Brand. Journal of Brand Management, 13, (4/5), 257.

Harris, F., \& De Chernatony, L. (2001). Corporate Branding and Corporate Brand Performance. European Journal of Marketing, 35(3/4), 411-456.

Hartley, J. F. (1994). Case Studies in Organizational Research. In C. Cassell \& G. Symon (Eds.), Qualitative Methods in Organizational Research: A Practical Guide. London: Sage Publications.

Haslam, S. A., \& Ellemers, N. (2005). Social Identity in Industrial and Organizational Psychology: Concepts, Controversies and Contributions. In G. P. Hodgkinson \& J. K. Ford (Eds.), International Review of Industrial and Organizational Psychology (Vol. 20, pp. 39-118). Chichester: John Wiley.

Haspeslagh, P., \& Jemison, D. (1991). Managing Acquisitions: Creating Value Through Corporate Renewal. New York: The Free Press.

Haunschild, P. R., Moreland, R. L., \& Murrell, A. J. (1994). Sources of Resistance to Mergers Between Groups. Journal of Applied Social Psychology, 24(13), 1150 1178.

Hogg, M. (2006). Social Identity Theory. In J. Burke (Ed.), Contemporary Social Psychological Theories (pp. 111-136). Stanford: Stanford University Press.

Hogg, M., \& Terry, D. (2000). Social Identity \& Self-Categorization Processes in Organizational Contexts. Academy of Management Review, 25 (1), 121-140.

Hogg, M. A., \& Abrams, D. (1988). Social Identifications: A Social Psychology of Intergroup Relations and Group Processes. London: Routledge.

Ind, N. (2004). Living the Brand: How to Transform Every Member of Your Organisation into a Brand Champion. London: Kogan Page.

Jemison, D., \& Sitkin, S. (1986). Corporate Acquisitions: A Process Perspective. Academy of Management Review, 11(1), 145-163.

Johnson, R. B., \& Onwuegbuzie, A. J. (2004). Mixed Methods Research: A Research Paradigm whose Time Has Come. Educational Researcher, 33(7), 14-26.

Katsioloudes, M. (2006). Strategic Management: Global Cultural Perspectives for Profit and Non-Profit Organizations. Oxford: Elsevier.

Keller, K. L. (2000). The Brand Report Card. Harvard Business Review, Reprint (Jan-Feb 2000). 
Kidder, L. (1981). Research Methods in Social Relations (4th ed.). Chicago: Holt, Rinehart and Winston, Inc.

King, D. R., Dalton, D. R., Daily, C. M., \& Covin, J. G. (2004). Meta-Analyses of PostAcquisition Performance: Indications of Unidentified Moderators. Strategic Management Journal, 25(2), 187 - 200.

Larsson, R., \& Finkelstein, S. (1999). Integrating Strategic, Organizational, and Human Resource Perspectives on Mergers and Acquisitions: A Case Survey of Synergy Realization. Organization Science, 10(1), 1-26.

Lenski, G. (1984). Power and Privilege: A Theory of Social Stratification. Chapel Hill: The University of North Carolina Press.

Lubatkin, M. (1983). Mergers and the Performance of the Acquiring Firm. The Academy of Management Review, 8(2), 218-225.

Mabry, L. (2008). Case Study in Social Research. In P. Alasuutari, L. Bickman \& J. Brannen (Eds.), The Sage Handbook of Social Research Methods. Trowbridge: Sage Publications.

Mael, F., \& Ashforth, B. E. (1992). Alumni and their Alma Mater: A Partial Test of the Reformulated Model of Organizational Identification. Journal of Organizational Behavior, 13, 103-123.

Mael, F. A., \& Ashforth , B. E. (1995). Loyal From Day One: Biodata, Organizational Identification, and Turnover Among Newcomers. Personnel Psychology, 48(2), $309-333$.

Marks, M. L., \& Mirvis, P. H. (2001). Making Mergers and Acquisitions Work: Strategic and Psychological Preparation. Academy of Management Executive, 15, 80-94.

Marshall, C., \& Rossman, G. B. (2006). Designing Qualitative Research (4th ed.). Thousand Oaks: Sage Publications

Melewar, T. C., Bassett, C., \& Simões, C. (2006). The Role of Communication and Visual Identity in Modern Organisations. Corporate Communications, 11(2), 138-147.

Melewar, T. C., \& Jenkins, E. (2002). Defining the Corporate Identity Construct. Corporate Reputation Review, 5(1), 76-90.

Meyer, K. E., \& Lieb-Doczy, E. (2003). Post-Acquisition Restructuring as Evolutionary Process. Journal of Management Studies, 40(2), 459-482.

Mitchell, C. (2002). Selling the Brand Inside. Harvard Business Review, 80(1), 88-101. 
Mottola, G. R., Bachman, B. A., Gaertner, S. L., \& Dovidio, J. F. (1997). How Groups Merge: The Effects of Merger Integration Patterns on Anticipated Commitment to the Merged Organization. Journal of Applied Social Psychology, 27, 1335-1358.

Mullen, B., Brown, R., \& Smith, C. (1992). Ingroup Bias as a Function of Salience, Relevance, and Status. European Journal of Social Psychology, 22(2), 103- 122.

Nandan, S. (2005). An Exploration of the Brand Identity-Brand Image Linkage: A Communications Perspective. Journal of Brand Management, 12(4), 264.

Napier, N. (1989). Mergers and Acquisitions, Human Resource Issues and Outcomes: A Review and Suggested Typology. Journal of Management Studies 26(3), 271-289.

O'Leary, Z. (2004). The Essential Guide to Doing Research. London: Sage Publications.

Olie, R. (1994). Shades of Culture and Institutions in International Mergers. Organization Studies, 15(3), 381-405.

Olins, W. (1995). The New Guide to Identity: How to Create and Sustain Change Through Managing Identity. Brookfield, VT: Design Council/Gower.

Pablo, A. (1994). Determinants of Acquisition Integration Level: A Decision-Making Perspective. Academy of Management Journal, 37(4), 803-836.

Patton, M. Q. (1990). Qualitative Evaluation and Research Methods. Newbury Park, CA: Sage Publications.

Peng, M. W. (2006). Global Strategy. Cincinnati: Thomson South-Western.

Perry, M. K., \& Porter, R. H. (1985). Oligopoly and the Incentive for Horizontal Merger. The American Economic Review, 75(1), 219-227.

Pincus, J. D., Robert, A. P. R., Rayfield, A. P. R., \& DeBonis, J. N. (1991). Transforming CEOs into Chief Communications Officer. Public Relations Journal, 47(11), 22.

Prichett, P. (1987). Making Mergers Work: A Guide to Managing Mergers and Acquisition. Chicago: Irwin.

Pruzan, P. (2001). Corporate Reputation: Image and Identity. Corporate Reputation Review, 4(1), 50.

Quinn Patton, M. (2002). Qualitative Research \& Evaluation Methods (3 ed.). Thousand Oaks: Sage Publications.

Ruspini, E. (2002). Introduction to Longitudinal Research. London: Routledge.

Salter, M. S., \& Weinhold, W. A. (1978). Diversification via Acquisition: Creating Value. Harvard Business Review, 56(4), 166-176. 
Schulsinger, F., Mednick, S. A., \& Knop, J. (Eds.). (1981). Longitudinal Research: Methods and Uses in Behavioural Science. Massachusetts: Martinus Nijhoff Publishing.

Schutt, R. K. (2006). Investigating the Social World: The Process and Practice of Research (5th ed.). Thousand Oaks: Sage Publications.

Schweiger, D. M., Ivancevich, J. M., \& Power, F. R. (1987). Executive Actions for Managing Human Resources Before and After Acquisition. Academy of Management Executive, 1(2), 127-138.

Scott, S. G., \& Lane, V. R. (2000). A Stakeholder Approach to Organizational Identity. The Academy of Management Review, 25(1), 43-62.

Searle, R. H., \& Ball, K. S. (2004). The Development of Trust and Distrust in a Merger. Journal of Managerial Psychology, 19(7), 708-721.

Seo, M., \& Hill, N. S. (2005). Understanding the Human Side of Merger and Acquisition. Journal of Applied Behavioural Science, 41(4), 422-443.

Shah, P. P. (2000). Network Destruction, the Structural Implications of Downsizing. Academy of Management Journal, 43(1), 101-113.

Shrivastava, P. (1986). Postmerger Integration. Journal of Business Strategy, 7(1), 65-76.

Spring, G. S. (1997). Case Study as a Research Method. Retrieved 10 May 2008 from http://www.gslis.utexas.edu/ ssoy/usesusers/1391d1b.htm

Stahl, G. K. (2006). Every Merger or Acquisition Promises to Create Value From Some Kind of Synergy. Financial Times, 3.

Stahl, G. K., \& Mendenhall, M. E. (2005). Mergers and Acquisitions: Managing Culture and Human Resources. Stanford: Stanford Business Books.

Stake, R. E. (2005). Qualitative Case Studies. In N. K. Denzin \& Y. S. Lincoln (Eds.), The Sage Handbook of Qualitative Research. Thousand Oaks: Sage Publications.

Stybel, L. (1986). After the Merger. New England Business, 67-68.

Sumner, W. G. (1906). Folkways. New York: Ginn.

Tajfel, H. (1974). Social Identity and Intergroup Behaviour. Social Science Information, 23, 65-93.

Tajfel, H. (1975). The Exit of Social Mobility and the Voice of Social Change: Notes on the Social Psychology of Intergroup Relations. Social Science Information, 14, 101-118.

Tajfel, H., Billig, M. G., Bundy, R. P., \& Flament, C. (1971). Social Categorization and Intergroup Behaviour. European Journal of Social Psychology, 1(2), 149-178. 
Tajfel, H., \& Turner, J. (1979). An Integrative Theory of Intergroup Conflict. In W. G. Austin \& S. Worchel (Eds.), The Social Psychology of Intergroup Relations. Monterey: Brooks/Cole.

Tajfel, H., \& Turner, J. C. (1986). The Social Identity Theory of Intergroup Behaviour. In S. Worchel \& W. G. Austin (Eds.), Psychology of Intergroup Relations (pp. 7-24). Chicago: Nelson-Hall.

Terry, D. J., \& Callan, V. J. (1998). In-group Bias in Response to an Organisational Merger (Vol. 2).

Terry, D. J., Carey, C. J., \& Callan, V. J. (2001). Employee Adjustment to an Organizational Merger: An Intergroup Perspective. Personality and Social Psychology Bulletin, 27(3), 267-280.

Terry, D. J., \& O'Brien, A. T. (2001). Status, Legitimacy, and Ingroup bias in the Context of an Organizational Merger Group Processes \& Intergroup Relations, 4(3), 271289.

Trautwein, F. (1990). Merger Motives and Merger Prescriptions. Strategic Management Journal, 11, 283-295.

Turner, J. C., Hogg, M. A., Oakes, P. J., Reicher, S. D., \& Wetherell, M. S. (1987). Rediscovering the Social Group: A Self-Categorization Theory. Oxford, England Blackwell.

Tyler, T. R. (2000). Social Justice: Outcome and Procedure. International Journal of Psychology, 35(2), 117 - 125.

Vaara, E. (2003). Post-Acquisition Integration as Sensemaking: Glimpses of Ambiguity, Confusion, Hypocrisy, and Politicization. Journal of Management Studies, 40(4), 859 - 894.

Van Knippenberg, D., Van Knippenberg, B., Monden, L., \& De Lima, F. (2002). Organizational Identification after a Merger: A Social Identity Perspective. The British Psychological Society, 41(2), 233-252.

Van Leeuwen, E., \& Van Knippenberg, D. (2003). Organizational Identification Following a Merger: The Importance of Agreeing to Differ. In S. A. Haslam, D. Van Knippenberg, M. Platow \& N. Ellemers (Eds.), Social Identity at Work: Developing Theory for Organizational Practice (pp. 205-221). Philadelphia: Psychology Press.

Van Leeuwen, E., Van Knippenberg, D., \& Ellemers, N. (2003). Continuing and Changing Group Identities: The Effects of Merging on Social Identification and Ingroup Bias. Personality and Social Psychology Bulletin, 29(6), 679-689. 
Van Oudenhoven, J. P., \& De Boer, T. (1995). Complementarily and Similarity of Partners in International Mergers. Basic and Applied Social Psychology, 17(3), 343 - 356.

Villinger, R. (1996). Post-Acquisition Managerial Learning in Central East Europe Organization Studies, 17(2), 181-206.

Yin, R. (2003). Case Study Research: Design and Methods. Thousand Oaks: Sage Publications.

Zaheer, S., Schomaker, M., \& Genc, M. (2003). Identity Versus Culture in Mergers of Equals. European Management Journal, 21(2), 185. 


\section{APPENDIX 1}

\section{Appendix 1: Interview schedule}

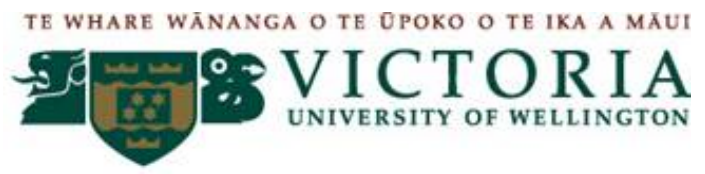

The Effects of Ingroup Development in a Post-merger Joint-Brand Context

\section{Interview Schedule}

Prescript: "Reflect on the communication that you receive around the office."

Question 1: Are there central concepts or ideas that are transmitted through this communication?

Prescript: "Reflect on the physical features of your office..."

Question 2: What does this communicate to you?

Prescript: "Reflect on the human dynamics of the collections area itself."

Question 3: Are there shared values and beliefs which exist? If so, what are they and in what ways do they exist?

Question 4: Is your working environment cohesive, or are there separations that exist, and why?

Prescript: "When change occurs in the bank..."

Question 5: How do you think the organisation treats employees? 
Question 6: What do you think have been the motives behind the changes, comment?

Question 7: How do you think the change process is managed?

Question 8: What is it about the collections unit that motivates you?

Question 9: What could be done to improve your motivation?

Question 10: What is it about the company or the collections unit that increases your commitment to the organisation or the role?

Question 11: What could be done to improve your commitment?

Question 12: What does it mean to be a member of Blue (or) Green collections?

Question 13: What is the difference between Blue and Green collections?

Prescript: "Within your role in the bank, there are three entities that you are involved with. The first is the overall company, Blue Green Bank. The second is the business unit, Consumer Finance Collections. The third is your team, Blue/Green Card/Personal Loan Collections..."

Question 14: Which entity or entities do you relate to more, and why?

Question 15: What is your idea of general best practice in service standards?

Question 16: How does the Blue (or) Green 'service promise' differ from typical best practice?

Question 17: To what extent do you implement this (service promise) in your work? 


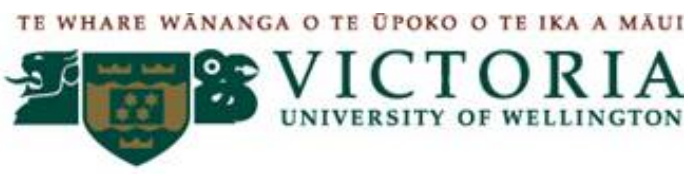

\section{The Effects of Ingroup Development in a Post-merger Joint-Brand Context}

\section{Information Sheet for Research Participants}

\section{Summary}

The research is looking at [bank's name] as an example of a post-acquisition environment. The project itself is focused on a change of strategy in the [bank's name] Collections Unit. The research is partially funded by [bank's name] who has given the researcher access to the Collections Unit within the Consumer Finance Division of the bank. Approval for the research has been obtained from the Victoria University Human Ethics Committee as well as [bank's name]. Data from the interviews will be used for an academic thesis, which will be summarised and presented to [bank's name], and may be used in further published articles.

\section{Research Process}

As a researcher I am looking for employees who are willing to share their views of the [bank's name] Collection Unit. I wish to draw on the vast and diverse experience of collection staff. A participant in the study will be involved in two interviews, the first to be conduced in mid 2007, and the second to be conducted in early 2008.

The interview questions are discussion based, which means the interviews will run in a conversational style. The interviews will be conducted during working hours, and are expected to last around thirty minutes each. Each interview will be tape recorded with the participant's consent. 


\section{Data and Participant Protection}

- The confidentially of each participant will be maintained. In any written material, names of the participants will be replaced with pseudonyms. A pseudonym may also be used in place of the [bank,s name].

- A summary of the interviews will be available to participants at least two weeks before the cut off period for withdraw from the study.

- The participants can withdraw themselves and their contribution to the research at anytime before the 29/02/2008.

- Interview data will be both electronically secured by a password protected computer, and physically secured in a padlocked storage unit.

- Interview data will be destroyed no later than three years after the conclusion of the research.

\section{The Researcher}

My name is Jay Newdick and I am an Honours graduate of Victoria University, Wellington. The research conducted at [bank's name] will contribute to my Masters in Commerce and Administration. The research is supervised by Dr. Sally Riad who is an experienced scholar in mergers and acquisitions.

Jay Newdick

Ph: 044636932

Work email: NewdickJ@anz.com
Sally Riad

Ph: 044635079

Work email: Sally.Riad@vuw.ac.nz 


\section{APPENDIX 3}

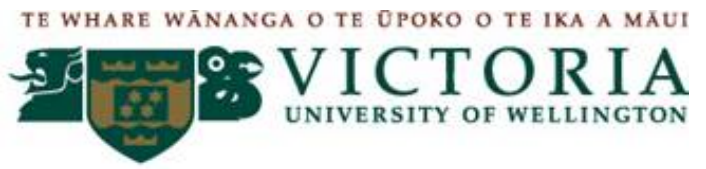

\section{The Effects of Ingroup Development in a Post-merger Joint-Brand Context}

\section{Participant Consent Form}

I have been informed of and understand the research project. This has been communicated through an information sheet, for which I have had the opportunity to question the research project and I am now satisfied with my understanding. I also understand that I may withdraw myself and all information from the study by the $29 / 02 / 2008$, without having to provide reason. I am aware that data will be secured electronically by password computer and physically by a padlocked storage unit until that point. Outside of the researcher and the research supervisors, I understand that all data is aggregated and pseudonyms are used to protect participant confidentiality. I am also aware that data may be used in an academic thesis, other published papers, and formal presentations. I also understand that data will be destroyed no later than three years from the conclusion of the research.

I agree to the use of a tape recorder in my interviews (tick if you agree)

I would like to receive a summary of findings before any data is publicly released (tick if you agree)

Any other conditions (please specify)

I have read and understand the agreement shown above and I agree to participate in this study. 


\section{Participant}

Name:

Date:

Signature:

\section{Researcher}

Name: $\quad$ Jay Newdick

Date:

Signature:

Contact Details

Researcher

Jay Newdick

Ph: 044636932

Work email: NewdickJ@anz.com
Primary Research Supervisor

Sally Riad

Ph: 044635079

Work email: Sally.Riad@vuw.ac.nz 